\title{
Element Specific Magnetometry Combining X-ray Circular with Linear Dichroism: Fundamentals and Applications
}

\author{
I.A. KOWALIK* \\ Institute of Physics, Polish Academy of Sciences, al. Lotników 32/46, 02-668 Warsaw, Poland \\ (Received October 31, 2014; revised version January 20, 2015; in final form March 12, 2015)
}

This article gives an overview of the use of X-ray absorption spectroscopy to characterize the magnetic properties for technologically important, low dimensional magnetic materials. An overview is given both for the experimental hardware, the measurements and the analysis of the spectra. The information obtained is discussed for metallic and semiconducting systems, using both the X-ray magnetic circular dichroism and the X-ray linear magnetic dichroism spectroscopy.

DOI: $10.12693 /$ APhysPolA.127.831

PACS: 78.70.Dm, 78.20.Ls, 75.50.Pp, 75.50.Tt

\section{Short historic overview of the XMCD technique}

To start this historical outline one should mention that although the X-ray magnetic circular dichroism (XMCD) technique is relatively young, the magneto-optical effect characteristic of the interaction between light and a magnetic medium was studied already in the nineteenth century, when several key discoveries were made enabling the development of new experimental techniques and thus studying so far unknown properties of matter. Let's start with Augustin Fresnel, who was the first one to envision the existence of circular and elliptical light polarization. In his equations on light waves he described how light polarized in circular or elliptical states can be produced from linearly polarized light by total internal reflection. The field of magneto-optics was initiated, according to documented materials, by Michael Faraday, who in 1845 discovered the phenomenon of the rotation of the polarization plane of the light transmitted through a medium located in an external magnetic field [1]. Today this effect is known as the Faraday effect. The application of a magnetic field to the medium through which linearly polarized light propagates, seen as the superposition of circularly left and right handed light, can lift the degeneracy of the circular state of the light polarization. Depending on the optical anisotropy of the material, the rotation of the polarization plane of the transmitted light can be observed.

The phenomenon of circular dichroism (CD) is defined as the difference in the absorption of circularly left handed versus right handed polarized components of the light for a given optically active (chiral) material. The French physicists Francois Arago and Jean-Baptiste Biot contributed to the discovery of the CD phenomenon. In the first half of the nineteenth century they did study the

*corresponding author; e-mail: ikowalik@ifpan.edu.pl polarization of the light and its interaction with matter and discovered the rotation of the vibration plane of the incident linearly polarized light, when light is transmitted through a parallel plate of crystalline quartz cut perpendicular to its optical axis. This effect was used later in the construction of ellipsometers. Finally, in 1896, again a French physicist, Aimé Cotton, studying the interaction of light with chiral molecules did observe differences in the absorption of the left handed versus the right handed circular light. However, substances not active optically (achiral) may also exhibit the phenomenon of circular dichroism if an external magnetic field is applied, because the magnetic field lifts the high degree of degeneracy due to the magnetic quantum number. This effect is known as the magnetic circular dichroism (MCD) effect. MCD occurs due to optical transitions under an applied external magnetic field, governed by selection rules in the quantum numbers, a phenomenon known since 1896 as the Zeeman effect. It results from the quantization of the magnetic moments of the electrons in the medium, as the electrons with spin parallel to the direction of the magnetic field are found in quantum states corresponding to a different energy as compared to the electrons with an antiparallel spin. Another magneto-optical effect was discovered in 1877. This is the magneto-optical Kerr effect (MOKE) in which polarized light is reflected at the surface of ferromagnetic material, with the magnetization vector oriented perpendicular or parallel to the surface.

At about the same time, in 1895, investigating the phenomenon of cathode rays Roentgen discovered invisible, high-energy radiation in the spectral range from ultraviolet to gamma rays. This discovery initiated research on the physical properties of X-ray radiation and its interaction with matter. Despite the fact that the discovery of $\mathrm{X}$-rays was made after the discovery of magneto-optical effects, it took still several decades for magneto-optics experiments based on X-rays.

The first theoretical predictions of a strong magnetic circular dichroism effect has been made in 1975 by E.A. 
Stern with collaborators, concerning the $M_{2,3}$-edges of ferromagnetic nickel in X-ray absorption, in a Faraday type of geometry $[2,3]$. The calculations were done using an approach based on the single particle model of optical transitions from a core state of prescribed symmetry into conduction band states with symmetry corresponding to allowed optical transitions. As the authors remarked, the absorption spectra obtained using circularly polarized X-ray light carry information on the electronic structure, including the spin-orbit splitting of the $3 p$ core states, the spin polarization of the empty $d$ states of the conduction band and the distribution of density of $d$-band states above the Fermi level [3]. In 1985, B. T. Thole with collaborators, within the framework of the simple atomic multiplet approach based on electric dipole selection rules for electronic transitions, predicted the existence of a strong X-ray linear magnetic dichroism (XLMD) at the $M_{4,5}$ absorption edges of rare earth elements [4]. The authors found that the polarization dependence of the absorption spectra at the $M_{4,5}$-edges of the magnetically coupled rare-earth ions depend on the relative orientation between the direction of the sample magnetization and electric field vector of the X-rays. The intensity of the XLMD effect is proportional to the square of the magnetization and allows to determine the local magnetic moment of an antiferromagnet, ferrimagnet or ferromagnet. A year later, in 1986, the theoretically predicted XMLD effect was measured experimentally by van der Laan and collaborators who studying the TbIG in the ACO synchrotrons laboratory in Orsay (France) did observe a strong magnetic linear dichroism effect at the $M_{4,5}$-edge of terbium [5]. In 1987 also magnetic circular dichroism was experimentally confirmed, which for the first time was observed at the $K$ absorption edge of iron in an Fe film $[6,7]$. These results were obtained by G. Schütz and collaborators, using elliptically polarized $\mathrm{X}$-rays from a hard X-ray beam line at the storage ring DORIS at DESY in Hamburg (Germany). It was the first successful X-ray absorption experiment using circularly polarized light, despite that the $K$-edges show a much weaker XMCD signal than the $L$-edges. The existence of magnetic circular dichroism in the X-ray domain was confirmed also by means of the first successful experiment using the spin-dependent extended X-ray absorption fine structure (magnetic EXAFS) technique, when the authors did observe an XMCD signal at the $K$-edge of Fe and $L$-edges of gadolinium in a metallic Gd sample and the ferromagnetic $\mathrm{Gd}_{3} \mathrm{Fe}_{5} \mathrm{O}_{12}$ compound [8].

An important step forward in the development of the XMCD technique was to broaden its application in the soft X-ray spectral range. In 1990 C.T. Chen with collaborators published the results obtained at the NSLS synchrotron radiation laboratory in Brookhaven (USA), where these authors did observe a very strong XMCD signal at the $L_{3,2}$-edge of ferromagnetic nickel [9]. As the authors summarized in their publication, the obtained results reflect the details of the electronic structure and magnetic structure of the studied materials. The strong
XMCD signal observed at the $L$-edge of nickel, which is about two orders of magnitude stronger than at the $K$-edge of transition metals, motivates the further studies using circularly polarized soft X-rays for magnetically ordered $3 d$ ferromagnets and $4 f$ rare earths systems [9]. As the authors show, by the comparison of the simultaneously measured spin independent and spin dependent absorption spectra one can distinguish between magnetic and non magnetic neighboring atoms.

Starting in the early 1990s the access to high photon flux X-ray absorption beam lines providing circular X-rays became easier by the early 2000's, with an increasing amount of synchrotron radiation facilites where the XMCD technique is widely used. A basic tool for the quantitative analysis and interpretation of XMCD experimental data was developed by B.T. Thole and P. Carra in 1993 [10, 11]. Using a single ion approximation the authors developed the so called magneto-optical sum rules, relating the integral of the dichroic spectral areas of the XMCD response to the ground state expectation value of the magnetic moment of the photoexcited atom. By applying these sum rules to the XMCD spectra we can determine both the spin [10] and orbital [11] magnetic moments on a per atom basis for the transition metals and the rare earths. The direct separation of the spin and orbital magnetic moments of the studied material remains to this day a unique advantage of the XMCD technique. The magnetic moments can be determined directly based on the sum rule parameters obtained from the integration of the XMCD spectra. The orbital sum rule states that the integral of the dichroic signal of the absorption edge over the spin orbit split edges for a $3 d$ ferromagnet, is directly proportional to the orbital part of the magnetic moment for the ground state of the atoms. The sum rules will be discussed in more detail in a later section of the article. The acronym MCD has been used for several techniques, such as magneto optical Kerr effect (MOKE), X-ray absorption spectroscopy (XAS) and X-ray photoemission spectroscopy (XPS). Finally, given the high number of publications in this area using X-ray absorption, it has been reserved for absorption spectroscopy only.

\section{Experimental hardware: requirements and description}

An experimental system for XMCD and XLMD spectroscopy measurements should satisfy a few basic requirements:

- allow the choice of circular left or circular right handed polarization of X-ray light (for XMCD). Alternatively allow the linear vertical or linear horizontal polarization of X-rays (for XLMD), and allow the rotation of the magnetic field direction, at least around the polar axis;

- allow the sample rotation at least around the polar axis, to choose the photon incidence direction versus the sample surface;

- allow to measure the absorption coefficient. For hard $\mathrm{X}$-rays this may be achieved in transmission geometry for 
thin samples. For soft X-rays, typically by measuring a secondary channel (for example in total electron yield or fluorescence yield) proportional to the absorption coefficient.

\subsection{X-ray sources and the polarization of the light}

Typically dedicated X-ray sources such as specially designed undulators or wigglers are used for the production of circularly polarized radiation of high brilliance and variable energy in the X-ray regime. In this article, we discuss in some detail, the example of an elliptically polarizing undulator (EPU), operating at the MAX IV synchrotron radiation laboratory, used for the production of soft X-rays. In this case almost purely circular X-rays can be produced. Elliptically polarized X-rays are also produced at bending magnets but with a lower brilliance. For a bending magnet X-ray source the radiation is linearly polarized in the plane of the electron orbit, and elliptically polarized light is obtained by a local deviation of the electron motion out of the plane by a small angle. Such a deviation of the beam out of the plane of the electron orbit is not the most efficient way to obtain circularly polarized light, as it increases the probability that intensity fluctuations may appear. To work with a high degree of light helicity one has to face also a substantial decrease of the photon flux, especially when one is working in the range of higher photon energy, above $1 \mathrm{keV}$. The EPU source allows to work both with a high photon flux and a high brilliance.

To keep the mathematical description of the polarization state of the X-ray beam as easy as possible, typically simplifying assumptions are made. It is assumed that the degree of the transverse and longitudinal coherence of the $\mathrm{X}$-rays is sufficient for the $\mathrm{X}$-rays to be described by the coherent superposition of two perpendicular components of the electric field as is also done in the optical range. These components can be phase shifted, allowing to describe linearly, circularly and elliptically polarized light. Below, this simple mathematical treatment is summarized, allowing to fix notations and give typical orders of magnitude for the various parameters.

A general expression for the electric field $\boldsymbol{E}$ of the $\mathrm{X}$-ray beam propagating in the $\mp z$ direction can be be written in terms of cosine function as

$$
\begin{aligned}
& \boldsymbol{E}(z, t)=E_{x}\left(\cos (\omega(t \mp z / c)+\Delta \varphi) \hat{e}_{x}\right. \\
& \quad+E_{y}\left(\cos \omega(t \mp z / c) \hat{e}_{y},\right.
\end{aligned}
$$

with $E_{x}$ and $E_{y}$ representing the amplitude of the electric field in the $x$ and $y$ direction, respectively. $\hat{e}_{x}$ and $\hat{e}_{y}$ are the unit vectors for the $O_{x}$ and $O_{y}$ axis in a referential system [12]. $\Delta \varphi$ is the relative phase shift between the two electric field components. The polarization state of a beam of light can be then described in terms of the Stokes parameters. These parameters are represented by four quantities, which are functions of observable quantities and which can be expressed as

$$
S_{0}=\left(E_{x}^{2}+E_{y}^{2}\right) / 2 N, \quad S_{1}=\left(E_{x}^{2}-E_{y}^{2}\right) / 2 N,
$$

$$
S_{2}=E_{x} E_{y} \cos (\Delta \varphi) / N, \quad S_{3}=E_{x} E_{y} \sin (\Delta \varphi) / N,
$$

where $N$ is a normalization factor. The first parameter $\left(S_{0}\right)$ gives the total intensity of the electric field. The $S_{1}$ and $S_{2}$ parameters give the degree of linearly polarized light in the horizontal plane and at $45^{\circ}$ versus the horizontal plane, respectively. The last parameter $\left(S_{3}\right)$ gives the degree of circularly polarized radiation. These Stokes parameters are related to each other through

$$
S_{0}^{2}=\frac{S_{1}^{2}+S_{2}^{2}+S_{3}^{2}}{P_{c}^{2}},
$$

where $0 \leq P_{c} \leq 1$ is a measure of the degree of circular polarization. $P_{c}=1$ characterizes fully circularly polarized light and $P_{c} \leq 1$ indicates a degree of partial polarization.

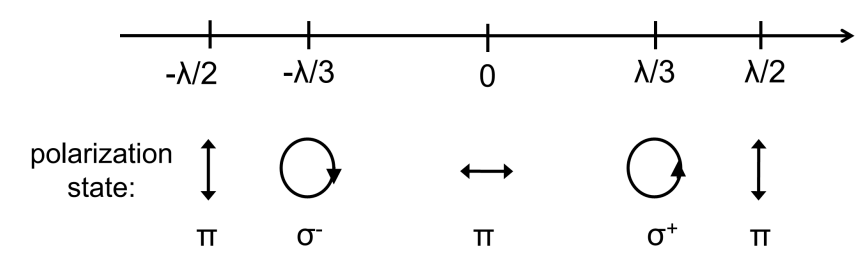

Fig. 1. Using an EPU the polarization state of the $\mathrm{X}$-rays can be varied. The EPU uses a periodic magnetic field of period $\lambda$, to influence the electron motion in the storage ring.

In the case of an undulator source, X-rays can be either circularly or linearly polarized. This is shown for the EPU design in Fig. 1 as one shifts the undulator magnet arrays. Both helicity states can be produced $(\Delta \varphi=\mp \varphi / 2)$. Also linear light in the electron orbit plane or perpendicular to it can be easily obtained. Typically for the variation of the polarization state of the $\mathrm{X}$-ray light only the undulator magnet arrays are shifted relative to each other (Fig. 1). This requires no motion of the source or some other beam line component. In the case of a bending magnet source an out of plane motion of the source can be performed, which is achieved by means of supplementary magnetic fields at the storage ring, which influence the motion of the electron bunches. Alternatively optical access to the source is partly shielded so that only the out of plane part of it is reaching the beam line optical elements. This solution allows for a high degree of flexibility, as the same beam line can be used for several types of experiments. In particular it was chosen for the first beam line of Solaris, the Polish National Synchrotron Radiation laboratory. For such a solution the degree of circular polarization is given below. A consequence of using the X-ray light source out of the storage ring plane is that a higher degree of circular polarization correlates with a lower photon flux. Using the in plane and out of plane components of the electric field of the radiation, the degree of circular polarization can be expressed as: 


$$
P_{c}=\frac{2 E_{\perp} E_{\|} \sin ^{2}(\Delta \phi)}{E_{\perp}^{2}+E_{\|}^{2}},
$$

were $\Delta \phi$ is the phase shift between the horizontal $\left(E_{\|}\right)$ and vertical $\left(E_{\perp}\right)$ E-fields. Assuming that $\Delta \phi=\pi / 2$, $I_{\|}=E_{\|}^{2}$ and $I_{\perp}=E_{\perp}^{2}$ the equation reduces to the more familiar form

$$
P_{c}=\frac{2 \sqrt{I_{\perp} I_{\|}}}{I_{\perp}+I_{\|}}
$$

and the degree of circular polarization can ideally be determined once the two orthogonal electric field components are determined.

There are several ways to characterize the degree of circular polarization of the X-ray beam, which is important for X-ray magnetic circular dichroism measurements in the X-ray absorption spectroscopy mode. A convenient possibility is provided by XMCD on magnetic materials characterized by magnetometry in the laboratory, where the magnetic state is known. If possible a single magnetic domain sample is used to facilitate the data analysis. Such magnetic standards can be permanently mounted at the beam line as typically Ultra High Vacuum is required for the beam line operation.

In practice, for a specific spectral range, to make a determination of the degree of circularity, a well known magnetic standard can be used, presenting well known absorption edges within the energy range of interest. For the soft X-ray regime the standard sample is usually a thin film of $\mathrm{Co}, \mathrm{Ni}$ or $\mathrm{Fe}$ where the atomic spin and orbital moments are previously determined by means of conventional magnetometry and if possible already by means of XMCD as well. An easy way to determine the degree of circular polarization is to calculate the atomic orbital and spin moments of the standard, following the well established magneto-optic XMCD sum rules. These magnetic moments add up to the magnetic moment measured in the laboratory. Often one relies on the known values of the spin and orbital moments from the XMCD literature assuming that the standard is of high magnetic quality.

In Fig. 2, as an example of a real system, is shown a schematic (a) and real (b) picture of the EPU operating at the I1011 beam line located at the MAX II ring $(1.5 \mathrm{GeV})$ at the MAX IV synchrotron radiation facility in Lund (Sweden). The undulator consists four movable jaws of permanent magnets arranged to alternate the magnetic field. This source provides soft X-rays in the energy range $100-2000 \mathrm{eV}$, allowing to study technologically relevant magnetic materials based on transition metal and rare earth atoms. By changing the phase between the individual jaws of the magnets one can choose any needed polarization state of the X-rays from linear (horizontal and vertical) to the circular polarization of the light. This makes possible not only XMCD measurements, but also XLMD. High photon flux allows for measurements even for diluted magnetic materials (with a magnetic ion concentration of less than $1 \%$ ).
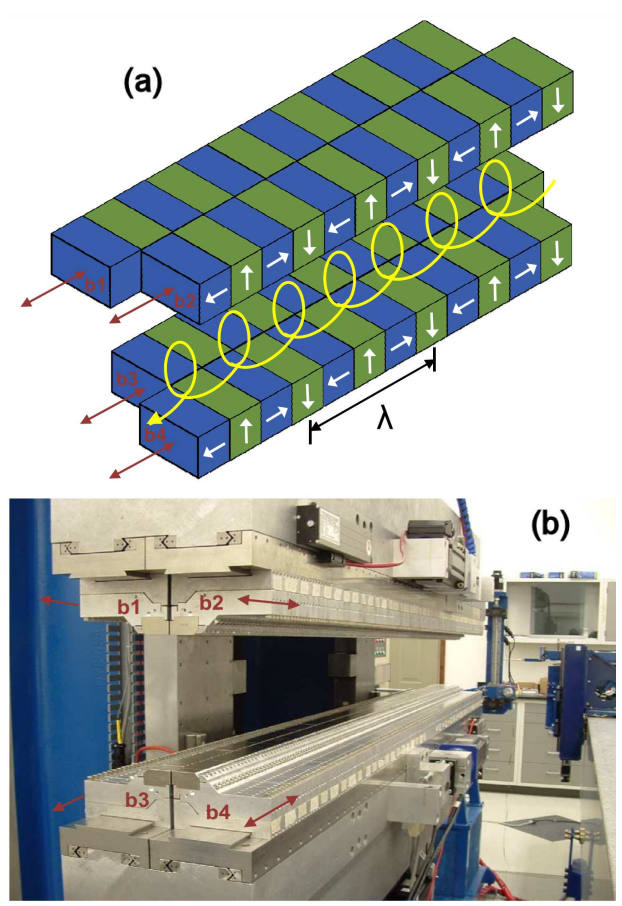

Fig. 2. A schematic (a) and real (b) picture of EPU operating at the I1011 beam line located at the MAX II ring $(1.5 \mathrm{GeV})$ at the MAX IV synchrotron radiation facility in Lund (Sweden).

\subsection{Layout of the beam line}

The experimental layout for XMCD and XLMD measurements will be discussed, as an example, for the I1011 beam line of the MAX II ring at the MAX IV synchrotron laboratory (Fig. 3). An important issue for magnetic measurements by means of the XMCD technique using synchrotron radiation is, among other things, the full control of the degree of polarization over the whole energy range covering the absorption edges of all relevant elements, in combination with good photon energy resolution and sufficient photon flux. For the end station a variable direction of the applied magnetic field vector is very useful, allowing for measurements in both the easy as well as the hard direction, without a sample rotation or any other type of sample motion. These aspects concerning new I1011 beam line will be described below.

As mentioned earlier, the I1011 beam line is based on the EPU source (Fig. 2). To optimize the photon flux and resolving power, the collimated plane grating monochromator (cPGM) is used. The monochromator contains three blazed gratings, which can be exchanged in vacuum: 336,1221 , or $1400 \mathrm{~mm}^{-1}$. A plane grating solution is used for the $\mathrm{X}$-ray monochromatization, which maintains the polarization state over the whole energy range covered by the monochromator. The possibility of selecting the so-called $c_{f f}$ parameter allows balancing between energy resolution, photon flux or higher order suppression. The cPGM is designed to deliver a resolution of 10000 over the whole energy range of operation, 


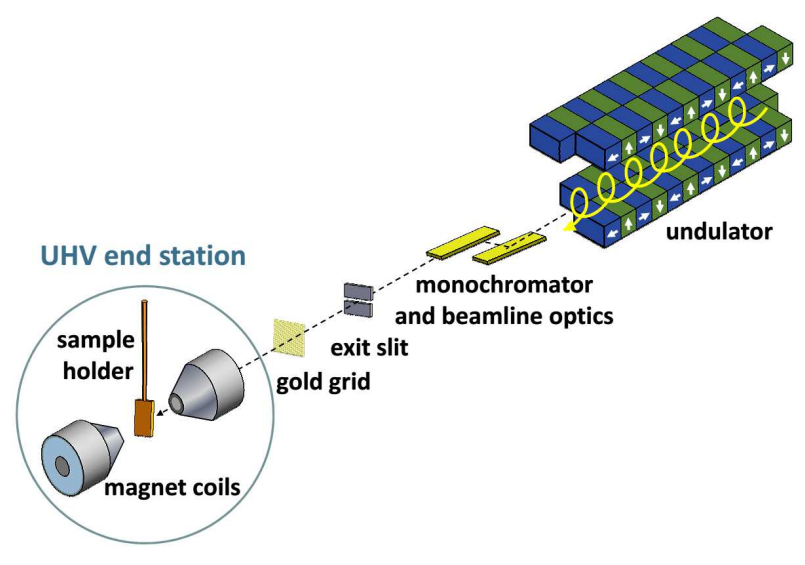

Fig. 3. Simple schematic representation of a typical beam line dedicated to XAS/XMCD measurements in the soft X-ray range. We show here the I1011 beam line at the MAX-IV synchrotron facility in Lund (Sweden). The beam line is equipped with an EPU, allowing for full control of the polarization state of the light. A collimated plane grating monochromator (cPGM from the BESTEC company) provides the high flux $\left(10^{11}-10^{12}\right.$ photons/sec) X-ray beam within the energy range of $0.1-1.7 \mathrm{keV}$. The reference signal $I_{0}$ is measured in the TEY mode using a gold mesh located between the exit slit and the end station.

but even with rather relaxed conditions $\left(c_{f f}=4.5\right.$, exit slit $60 \mu \mathrm{m})$ the resolving power is found to be more than 9000 for $\mathrm{N}_{2}$ and 6000 for Ne gas, while the flux at the sample is about $10^{11}$ photons/sec. The resolving power, photon flux and the size of the beam spot on the sample can be optimized by moving baffles in the beam path in both horizontal and vertical directions and changing the exit slit opening. For beam line I1011 the beam is focused onto the exit slit to maximize the energy resolution. The spot size of the diverging beam, after the exit slit, into the experimental station at the sample position is about $1 \mathrm{~mm}$ both in the vertical and horizontal directions. To monitor the reference signal $I_{0}$ the photocurrent from a fine electroformed Au mesh in the beam path is measured. The measurements are done in the photo ion yield mode.

The beam line is equipped with two end stations, which can be exchanged, depending on the experimental conditions needed for certain experiments. As an example in Fig. 4 the "octupole" magnetic UHV end station is shown. The name of the station finds its origin from the eight water-cooled electromagnets, spaced equidistantly over the surface of a sphere, which allows the application of the magnetic field in any direction. This solution, together with the possibility to rotate the whole chamber around the beam axis, allows to measure the magnetic properties with a variable geometry of applied magnetic field, electromagnetic radiation and sample orientation. These high current electro-magnets (100 A each) generate a field in the sample space of up to $1 \mathrm{~T}$. Such possibilities allow to optimize the measuring conditions

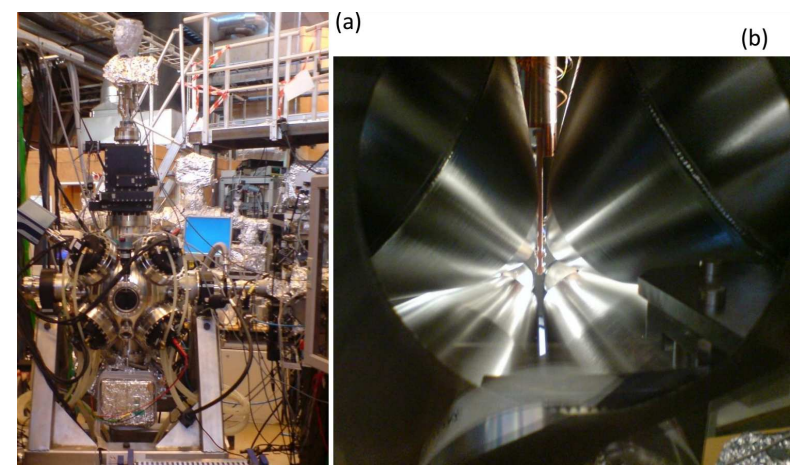

Fig. 4. Photographs of the "octupole" ultra high vacuum (UHV) end station located at the I1011 beam line at the MAX-IV synchrotron facility (Lund, Sweden). The UHV chamber is equipped with eight water cooled electromagnets generating a magnetic field in the sample space of up to $1 \mathrm{~T}(\mathrm{a})$. The sample positioned in the center between the electromagnets can be rotated around the polar axis (b). The whole chamber can be rotated around the beam axis by $\pm 90^{\circ}$, what allows to measure the magnetic properties with a variable geometry of applied magnetic field, electromagnetic radiation and sample orientation, also for the reflected X-ray beam from the sample at grazing X-ray angles.

for various magnetic systems studied by the XMCD and XLMD method. In another end station dedicated to surface science experiments, a rotatable set of coils has been installed. Here the applied magnetic field can only be rotated in the horizontal plane, by rotating the coils independently of the sample rotation. This system can be baked, allowing for ultra-high vacuum (Fig. 3). Only smaller magnetic field values can be reached, given the size limitations of the coils.

\section{X-ray Absorption spectroscopy with a magnetic contrast}

\subsection{Depth probing in XMCD and XLMD measurements in the total electron yield mode}

The incident radiation, here X-ray photons, is sufficiently energetic, so that even in the soft $\mathrm{X}$-ray regime it penetrates deeply into the solid, typically two to three orders of magnitude beyond the escape depth for the characteristic secondary electrons, $\lambda$. The electron escape depth is limited by the electron mean free path, which is the average distance traveled by an electron in between elastic or inelastic collisions. The mean free path depends on the kinetic energy of the electron and can be described by a universal curve for many solid materials. At very low kinetic energy $\left(E_{\text {kin }}\right.$ of a few $\left.\mathrm{eV}\right)$ the electrons have a long mean free path and should in principle be able to escape the sample, but since they have very low kinetic energy these electrons will not be able to overcome the work function. Therefore, by collecting the total electron yield after photon excitation, the electron escape depth one measures for the $L$-edges of the late $3 d$ elements ( $\mathrm{Fe}$, $\mathrm{Co}, \mathrm{Ni}$ ) is relatively short, about $17 \AA$. Due to the finite electron escape depth of the secondary electrons, in the 
total electron yield mode, one mainly probes the near surface region of the sample [13]. Using the value of the electron escape depth, an expression for the total electron yield, $Y$, versus photon energy, $E$, can be given for normal $\mathrm{X}$-ray incidence on the surface (wave vector perpendicular to the sample surface plane), as

$$
Y(E) \propto \int_{0}^{\infty} I(x, E) \mu(E) \mathrm{e}^{-\frac{x}{\lambda}} \mathrm{d} x,
$$

where $x$ is the depth away from the surface, where the absorption process takes place, $\mu(E)$ the photon absorption coefficient and $\lambda$ the electron escape depth. $I(x, E)$ represents the intensity of the X-rays at a certain photon energy, $E$, at the depth, $x$, and is expressed as

$$
I(x, E)=I_{0}(E) \mathrm{e}^{-x \mu(E)},
$$

where $I_{0}(E)$ is the incident $\mathrm{X}$-ray intensity. However, although the incident photons penetrate several hundred Angströms deep into the sample for soft X-rays, the number of electrons that can escape from the surface decreases exponentially with the depth away from the surface (Fig. 5).

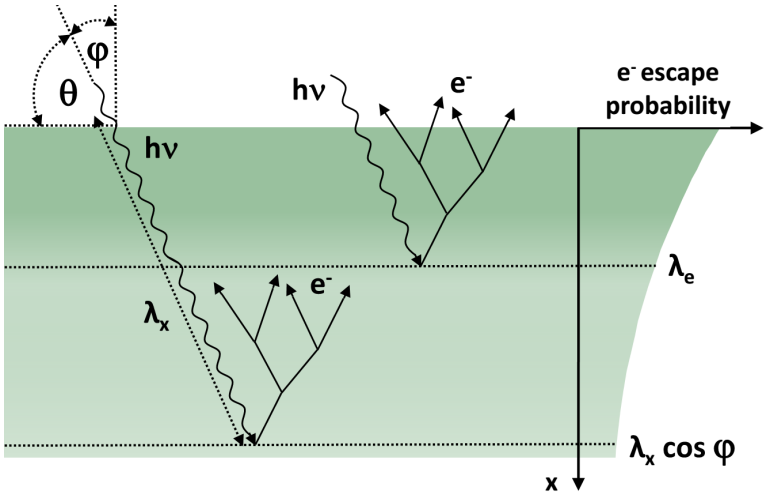

Fig. 5. Schematic visualization of the electron escape depth, $\lambda$, in XAS measured by means of the electron yield. The emitted electrons from depth, $x$, below the sample surface, reach the surface of the sample with a probability proportional to $\mathrm{e}^{-x / \lambda}$. The photons have a much larger penetration depth. XAS in TEY mode probes mainly the surface region of the sample.

The physical origin of a finite electron escape depth is the inelastic scattering of the electrons. Successive inelastic scattering events result in an electron cascade, where multiple low energy electrons are produced from a single high energy electron, created for example through the Auger process after photon absorption. Eventually, when the multiple low energy electrons have a kinetic energy lower that the work function of the sample, the low kinetic electrons of the cascade cannot escape from the sample surface, leading to a finite electron escape depth.

\subsection{Saturation effects in the electron yield}

In most cases the photon penetration depth, $\tilde{\lambda}$, is much larger than the electron escape depth, $\lambda$. However, if the condition $\tilde{\lambda} \gg \lambda$ is not fulfilled, the measured to- tal electron yield, $Y$, is not any longer proportional to the absorption coefficient, $\mu$, but the intensity of the most intense spectral features is suppressed or "saturated". When we observe a strong variation in $\mu$, for example in the vicinity of the $L_{3,2}$ peaks, compared to the absorption coefficient intensity at other energies, then measurements at low angles do not fulfill the condition $\tilde{\lambda} \gg \lambda$, which might lead to saturation effects in the vicinity of the peaks. Therefore, a corrective procedure to compensate for this effect is needed, which is presented here in the simple case of the half infinite solid. It can be shown that the electron yield for a half infinite solid can be described as:

$$
Y(E)=\mu(E) /\left(\mu(E)+\frac{1}{\lambda} \sin \theta\right),
$$

where $\theta$ is the $\mathrm{X}$-ray incidence angle relative to the sample surface. The normalized yield, $Y_{\text {norm }}$, is scaled to the experimental yield, $Y_{\text {exp }}$, using two constants $a$ and $b$ related as the equation:

$$
Y_{\text {norm }}=a+b Y_{\text {exp }} \text {. }
$$

From here the correction is done in four steps. From Eq. (2) we construct two equations: one for the preedge and one for the post-edge. We introduce the terms: $Y_{\text {norm }}^{\text {pre }}, Y_{\text {norm }}^{\text {post }}, Y_{\text {exp }}^{\text {pre }}$ and $Y_{\text {exp }}^{\text {post }}$. The first two terms are obtained from tables, e.g., the CXRO homepage [14], and the latter two terms are obtained from the XAS measurement. Using Eq. (2) and these terms the constants $a$ and $b$ are calculated. We now focus on another energy, e.g., the $L_{3}$ peak maximum and calculate $Y_{\text {norm }}^{L_{3}}$ from Eq. (2) using the above determined constants $a$ and $b$. As $Y_{\exp }$ we use the measured intensity at the $L_{3}$ peak, obtained from the same spectra as $Y_{\exp }^{\text {pre }}$ and $Y_{\exp }^{\text {post }}$. Using Eq. (1) with $Y(E)=Y_{\text {norm }}^{L_{3}}$ and with $\theta$ the X-ray incidence angle, the corrected $\mu$ is obtained. Finally, the corrected yield, $Y_{L_{3}}^{\text {corr }}$, is obtained using the corrected $\mu$ in Eq. (1) with $\theta$ set to $90^{\circ}$. It should be noticed that the procedure described above only gives the correction at one energy point, meaning that the corrective procedure has to be repeated for all energy points in the XAS spectra, point per point. More details on the possible occurrence of saturation effects in the electron yield and a corrective procedure can be found in the literature [15].

\subsection{Introduction to X-ray magnetic dichroism}

XMCD spectroscopy has many unique features, which can be exploited in particular in the case of low dimensional or dilute systems.

(I) Element specificity. By varying the photon energy, to match transitions at a particular atomic core level, one is sensitive to the magnetic moment of the specific photoexcited ionic core.

(II) A high degree of sensitivity due to the strong interaction of X-ray photons with the atom electron shells, making the technique ideal for the study, for example, of ferromagnetic surfaces or ferromagnetic nanodot arrays. Under applied magnetic field also magnetic systems with a weak magnetic response can be studied. 
(III) The possibility for vector information. The intensity of the dichroic difference is proportional to the projection of the sample magnetization on the X-ray propagation direction, or equivalently the wave vector of the X-ray beam.

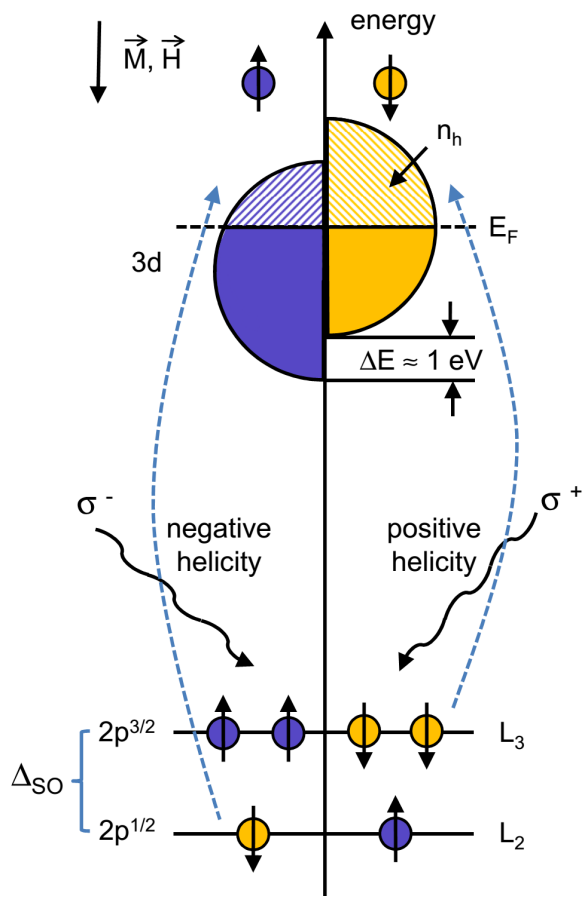

Fig. 6. Schematic diagram to describe the XMCD effect. The final states shown here are for a $3 d$ ferromagnet within a simple itinerant description for the $3 d$ states.

The X-ray magnetic circular dichroism technique allows for the independent determination of the values and the direction of the spin and orbital magnetic moments for each element of the material separately, on a per atom basis. Important about this technique is the high sensitivity it provides in order to study very dilute samples. This enables, for example, studies of ultra thin magnetic films and two dimensional arrays of magnetic nanostructured samples. The technique is based on the spin selective photon excitation of the electronic core levels of various atoms. For sizeable XMCD effects one tries to access initial states which have a sizeable spin-orbit energy. Using circularly polarized X-rays, for the absorption process, propagating along the direction of the macroscopic magnetization, the angular momentum of the photon $+\hbar$ or $-\hbar$, depending on the light helicity chosen, is transferred to the sample once a photon is absorbed [16, 17]. This leads to the vector magnetometry character of the XMCD technique, the dichroic intensity being proportional to $\boldsymbol{k} \cdot \boldsymbol{M}$, where $\boldsymbol{k}$ is the wave vector direction and $\boldsymbol{M}$ is the magnetization of the sample. The photon cannot couple directly with the spin of an electron without a finite spin-orbit energy, through which the photon angular momentum is transferred to the sample. As an example, the dipole selection rules in the case of the transition metal series allow for transitions from the $2 p_{3 / 2,1 / 2}$ initial states to the empty $3 d$ levels, with spin selectivity (Fig. 6). The unbalance of the empty $3 d$ final states, caused by the exchange splitting, leads to a spin selective absorption process and yields information about the spin polarization of the $3 d$ states. In a simplified itinerant electron picture, the spin moment $\left(m_{s}\right)$ is then directly related to the difference between the number of spin up $\left(N_{+}\right)$and spin down $\left(N_{-}\right) 3 d$ holes, reflected in the intensity of the $2 p_{3 / 2,1 / 2}$ "white lines" [16].

To describe XMCD one can use the "two step model" in a single electron picture (Fig. 6). In this simple picture of initial and final states, coupled by the dipole selection rules, as no spin flip is allowed in the absorption process, the transfer of angular momentum directly dictates the transition probability to the spin up or down final states, depending on the light helicity. The absorption process is discussed then in a two step process with an internal source of spin polarized electrons and a spin detector at the location of the atom, where the absorption process takes place.

The first step in the "two step model" is the process of absorption. The electron excitation, following the impact of the photon within the atom, is described through the dipole selection rule. The selection rules, which govern the electronic transitions, represent the overall conservation of angular momentum of the total system and one obtains $\Delta l= \pm 1$ due to the absorption of the photon, which is carrying an angular momentum of $\pm \hbar$. However, in the process of absorption of circularly polarized light one needs to fullfill an additional selection rule $\Delta m_{l}= \pm 1$, where the positive and negative sign of $m_{l}$ depends on the helicity of the incident of light. In this context, the photon absorption process in terms of the photon helicity contribution can be described by the equation:

$$
I^{ \pm}=I_{0}^{ \pm} \cdot \mathrm{e}^{-\mu^{ \pm} x}
$$

where $\mu^{+}$and $\mu^{-}$symbolize respectively the absorption coefficient for the right handed and left handed circularly polarized X-rays. In the second step of the "two step model", the imbalance of empty $3 d$ final states in a $3 d$ transition metal available for the two opposite spin states, leads to the imbalance in the number of final states involving the electron spin, thereby carrying the information about the spin polarization of the final $d$ states.

The XMCD difference, defined as the difference $\mu^{+}-$ $\mu^{-}$, is the intensity difference of the normalized X-ray absorption spectra to the atomic cross section, taken with a parallel and antiparallel orientation of the magnetization of the sample versus the photon spin. The XMCD difference is directly proportional to the strength of the atomic magnetic moment, but also depends on the relative orientation of the magnetization vector versus the wave vector direction of the incident light [16]. Dichroic contrast for the $3 d$ transition metals is predominantly 
seen around the spectral areas, where the "white lines" are observed in the absorption spectra.

Historically, within the band electronic model of itinerant electronic states, the magnetic properties of materials were described in the context of the occupied electronic states. Nowadays, due to the development of techniques based on electron transfer to the unoccupied electronic states and the detection of intensity asymmetries linked with these empty states, for techniques such as inverse photoemission or XMCD, more and more attention is paid to the unoccupied electronic states. In the simplest possible model the electron bands are represented as a parabolic distribution of states, which reflects the dispersion of the itinerant electrons in a solid. At a temperature $T=0$, all states located energetically below the Fermi energy are occupied and all the above are unoccupied electronic states.

Without a magnetic field, sub-bands for each of the spin directions are equally filled. An applied magnetic field shifts the energy of the sub-bands of parallel and antiparallel spins versus the direction of the field, in order to minimize the total energy. In this picture, as a result of this spin splitting, there appears a resultant nonzero spin magnetic moment. Such a model requires an external magnetic field. However, in the case of the $3 d$ transition ferromagnets, we have to deal also with the internal magnetic field, which Stoner took into account in the itinerant description of magnetism. The existence of this internal field causes the energy splitting of electron spin states in the two states oriented parallel (up) and antiparallel (down) versus the direction of the molecular field. In the Stoner model for ferromagnetism the itinerant electron imbalance of empty $3 d$ states available for spins up and spins down, due to their, on average, energy splitting, $\Delta E$, leads to the spin selectivity of to the absorption process and carries information about the spin polarization of the final $3 d$ states. The exchange splitting of the bands can be expressed by means of the phenomenological equation, as proposed by Stoner:

$$
\Delta E=-\frac{1}{4}\left(N_{+}-N_{-}\right)^{2} .
$$

For the transition metals this energy is about $1 \mathrm{eV}$.

In the itinerant view of magnetism the spin magnetic moment, $m_{s}$, is obtained as the difference between the number of majority $\left(N_{+}\right)$and minority $\left(N_{-}\right)$spins $1 / 2$ states:

$$
m_{s}=\left(N_{+}-N_{-}\right) \mu_{\mathrm{B}} .
$$

As the total number of $3 d$ states is fixed, the spin moment is also proportional to the difference in the spin up and spin down $3 d$ hole states. This last quantity is reflected experimentally in the intensity of the so called $2 p_{3 / 2,1 / 2}$ "white lines" in the XAS spectra, which is indeed mapping the $3 d$ density of final states, neglecting effects due to the core hole creation. To calculate the spin moment it appears therefore appropriate to integrate the XMCD difference spectra to obtain their spectral areas versus the photon energy.
The previous description captures the fact that an integral magneto-optic sum rule is needed to interpret the XMCD difference spectra, but fails to describe the exact details of this procedure starting from the atomic quantum numbers. On the other extreme one finds in the literature magneto-optic sum rules, where one uses linear combinations of the integrals of the "white line" intensity over the photon energy, derived within an atomic model. Trying to estimate how much one can hope to describe the magnetic properties of the sample, one notes that both these approaches do not explicitly involve the temperature dependence of the magnetic moment. On the other hand, one interesting feature of the XMCD technique is that not only the spin, but also the orbital moment of the photoexcited atom can be directly determined by means of magneto-optic sum rules from the XMCD spectra. To observe a finite orbital magnetic moment, one must assume symmetry breaking in terms of the electronic motion, so that the orbital motion of electrons within the solid is anisotropic on average. In a magnetic material such symmetry breaking has implications due to the spin-orbit $(S O)$ interaction. The $S O$ energy couples the spin and orbital moments, and favors the magnetic moment to lie along specific crystallographic directions. With consideration of the impact of the SO interaction one can explain magnetic phenomena such as the magneto-crystalline anisotropy, the anisotropic magnetoresistance and magnetostriction. The existence of a $S O$ energy for electronic states makes that the spin moment is tied to the crystal lattice. In relationship with this energy in the material there appear easy magnetization directions. In a localized atomic picture this interaction depends on the symmetry of the electric field due to the ligand atoms and the strength of the spinorbital coupling. More specifically, the orbital magnetic moment, $m_{l}$, linked to the $d$ electronic states is given by

$$
m_{l}=-\frac{\mu_{\mathrm{B}}}{\hbar}\left\langle L_{z}\right\rangle
$$

where the orbital moment average, $\left\langle L_{z}\right\rangle$, along the quantization direction can be calculated in second order perturbation theory. This calculation involves the sum of the matrix elements

$$
\left\langle\psi(\boldsymbol{k})\left|\hat{L_{z}}\right| \Phi_{n}\right\rangle,
$$

through all the empty electron states $\psi(\boldsymbol{k}) . \Phi_{n}$ stands for the ground state wave function of the system in this perturbative approach. Because the energy levels $2 p_{3 / 2}$ and $2 p_{1 / 2}$ are characterized by the opposite sign of spinorbital coupling, respectively $l+s$ and $l-s$, the spin polarization obtained for circularly polarized X-rays on the two edges $\left(L_{3}\right.$ and $\left.L_{2}\right)$ has the opposite sign. The anisotropy of the orbital magnetic moment is closely related with the magneto-crystalline anisotropy in a material. It has often been found that the orbital magnetic moment is larger in the direction of easy magnetization, and the difference between easy and hard magnetic direction is proportional the the magneto-crystalline anisotropy. For a uniaxial magnetic anisotropy system 
Bruno [18] derives in a simple perturbative approach an equation for the magneto-crystalline anisotropy:

$$
\Delta E_{s o}=E_{s o}^{\perp}-E_{s o}^{\|}=-\frac{\xi}{4 \mu_{\mathrm{B}}}\left(m_{l}^{\perp}-m_{l}^{\|}\right),
$$

where the constant $\xi$ is the atomic spin-orbit energy of the electronic valence states, linked with the existence of the magnetization and is of the order of $\sim 0.05 \mathrm{eV}$, where $m_{l}^{\perp}$ and $m_{l}^{\|}$are the values of the orbital moment along the magnetic hard and easy directions, respectively. This concept has been verified experimentally by angledependent XMCD measurements [19]. We conclude by noticing that the XMCD technique characterizes directly a quantity important for magnetism applications.

The temperature dependence in a magnetic system can also be observed by means of XMCD. In a simple meanfield Curie-Weiss model of a ferromagnetic system a spontaneous magnetization appears below the critical temperature, $T_{C}$, and the temperature dependence of the magnetic susceptibility above this temperature is described by the Curie-Weiss law

$$
\chi=\frac{N \mu^{2}}{3 k_{\mathrm{B}}} \frac{1}{T-T_{C}},
$$

where $N$ is the atom concentration, $\mu$ is the total magnetic moment of a magnetic atom, and $k_{\mathrm{B}}$ is the Boltzmann constant. To observe a finite XMCD signal, a finite projection of the magnetization is needed along the propagation direction of the X-ray beam. Only with the application of very high magnetic fields one may obtain a finite macroscopic projection of the magnetic moment to apply the magneto-optic XMCD rules in the paramagnetic regime, above the Curie temperature. It has been found for several systems that the Curie temperature can be traced also by means of the XMCD technique. Also the magnetic susceptibility can be measured by means of the XMCD technique, using a low frequency applied external oscillatory magnetic field [20]. At higher frequencies the XMCD technique can still be applied, allowing for element specific magnetization dynamics in the femtosecond range [21].

\subsection{Sum rules and data interpretation}

One can claim, that by means of some complicated experiments, without the use of XMCD, insight on the properties (I)-(III) defined in chapter 3.3 can be gained, although in a much more indirect manner, in particular in the case of (I). What makes, however, the XMCD technique even more appealing to use, is the possibility for a quantitative analysis of the dichroic spectral areas, by means of the magneto-optic sum rules. By the quantitative analysis of the dichroic spectral areas one retains properties (I)-(III), but obtains also not only the value of the spin moment for a specific ionic core, but also the value of the orbital moment. Here, following the normalization of the dichroic spectra, but also the proper procedures for spectral integration versus photon energy, the magnetic moment is obtained on Bohr magnetons per photoexcited atom. Several review articles have been presented in the literature on this topic $[22,23]$. Here we want to focus on the practical "hands on" applicability of the sum rules, and give some practical instructions, in particular for the soft X-ray domain, where the use of the sum rules is very widespread. We also want to focus on some of the possible typical pitfalls and mention some of the limitations and open questions in the literature.

Sum rules for specific spectral areas exist in several areas of spectroscopy, not least the optical regime. For the core levels of interest here, in the framework of the dipole approximation, it is straightforward to obtain a set of simple sum rules, by neglecting the rearrangements in the electronic structure, caused by the existence of a core hole in the final state. As in the case of Extended X-ray absorption fine structure (EXAFS), from a practical point of view it is best to use the sum rules for a relative comparison between a "standard" and the sample under investigation, the "unknown" material, whose spin and orbital moments should be determined versus the ones of the "standard". Below, following a similar logic of "relative analysis", the sum rules used for the $L$-edges of the $3 d$ transition metals are given

$$
\begin{aligned}
& m_{s_{\text {eff }}}=m_{s}-\frac{7}{2}\left\langle T_{z}\right\rangle=-C \frac{3 p-2 q}{r}, \\
& m_{l}=-C \frac{2 q}{3 r} \\
& \frac{m_{l}}{m_{s_{\text {eff }}}}=2 /\left(9 \frac{p}{q}-6\right),
\end{aligned}
$$

where $p$ i $q$ are values obtained from the integral of the dichroic difference spectrum, and $r$ is a value from the integral of halfsum (Fig. 8). The constant $C$ contains information on the degree of circular polarization of the $\mathrm{X}$-rays and the number of empty $3 d$ states $\left(n_{d}\right)$.

The number of $3 d$ holes can be determined to a very good approximation using the half sum of the minority and majority spectra. As a starting point one can refer to the number of empty $3 d$ states obtained from theory or an experimental standard. On the basis of the number of $3 d$ holes to be used to individual atoms in the material, based on theoretical calculations, one can start with a number of $0.83 d$ holes for bulk $\mathrm{Cu}$. Then one adds one more hole by decreasing the atomic number by one unit for each $3 d$ transition element. That way one obtains 3.8 holes in the case of bulk Fe [24]. The number of $3 d$ holes varies, depending on the local atomic environment and structure, but using a standard material during the XMCD measurements, one can easily estimate the value of the $3 d$ holes for the unknown material. The degree of polarization of the incident radiation is characteristic of the specific beam line, where the measurement is done. For the spin sum rule there is a corrective factor involving the local magnetic dipole operator, $\left\langle T_{z}\right\rangle$. This factor may be important for low symmetry environments. If this factor is not taken into account, one obtains an apparently anisotropic spin moment, which is a consequence of the anisotropy of the charge distribution around the atom. In this case, one should perform several measurements 
with the magnetic moment along both the easy and hard directions of the magnetic sample under investigation. For materials with high (cubic) symmetry of the lattice $\left\langle T_{z}\right\rangle \equiv 0,\left\langle T_{z}\right\rangle \neq 0$ in the case of materials with lower symmetry. For monocrystalline materials $\left\langle T_{z}\right\rangle$ can be determined on the basis of angle-dependent measurements, where the spin is oriented along at least two different crystallographic directions. The value of the orbital moment, $m_{l}$, is dependent only on the parameters $p$ and $q$, and is not affected by any corrections relating with the dipole operator. Therefore the orbital moment appears as a very robust quantity to be determined experimentally by means of the XMCD sum rules. Still the angular dependence is also needed, as the orbital moment is not isotropic, leading to the magneto-crystalline anisotropy.

The particular analytical form of the sum rules depends on the specific edge. For the sum rules given here, valid for the $2 p$ initial states of the $3 d$ transition metals, one sums over the dichroic areas of both the spin-orbit split $2 p$ states. The constants given in Eqs. (3), (4) express in a convenient manner the fact that the sum rule analysis is most accurate in a comparison versus a known "standard" compound. For the standard, such as bulk Ni, $\mathrm{Fe}$ or $\mathrm{Co}$, the number of $3 d$ final states, spin and orbital moments are known. One can determine then experimentally the constants in the mathematical expressions, given above, for the sum rules at the same beam line, under similar experimental conditions for the various beam line parameters. In a second step, using the same experimental setup and procedure to determine the spectral areas, one can determine then the number of the $3 d$ final states, the orbital moment and the spin moment of the "unknown" compound. If the spectra are normalized properly for the amount of atoms probed, the orbital and spin moments per photoexcited atom are determined. Such a per atom normalization can be obtained after normalizing the spectra to the high energy atomic continuum. To obtain the correct values of the magnetic moments also the finite degree of helicity of the circular $\mathrm{X}$-rays, as well as the angle between the sample magnetization and the direction of X-ray incidence, should be taken into account.

Such an "empirical" form of the sum rules, as given above, may appear to limit their use, as in principle it has been claimed that by means of these sum rules, an absolute determination of the spin and orbital moments is possible, once the number of the final states is known. In this context, it is necessary to note that there are also approximations made in order to obtain these sum rules in presented above simple form. However, the existence of the core hole in the final state cannot be taken into account easily. This affects the form of the atomic step, fitted to the spectra in order to obtain a spectral area proportional to the number of the final states [25]. There appears therefore a degree of arbitrariness in the moment determination by applying the sum rules, at least as far as the number of final state counting is concerned. The use of the sum rules for a relative analysis, between a mag- netic "standard" and an "unknown", minimizes the errors as core hole relaxation effects should have a similar impact on the spectra, if the "standard" is chosen carefully to match some of the properties of the "unknown".

A relative analysis allows to also minimize errors, which can be seen as due to an empirical and rather arbitrary character, when the sum rules are applied versus their theoretical form. As an example one can give the rather arbitrary limits of integration. Often in an experiment the spectral range is limited in both the pre-edge and post-edge regions. A relative analysis minimizes the errors, if the same limits are chosen. Also the particular spectral function of the X-ray monochromator and source used may influence the shape of the background function, which also strongly depends on the choice of the secondary channel used. For most practical applications in the soft X-ray regime, the spectra cannot be taken in the transmission mode, electron or fluorescence yield are used instead.

Several review articles have highlighted already the potential pitfalls and practical difficulties using the sum rules [25-27]. These have been applied extensively over the last two decades, in particular to study $\mathrm{Fe}, \mathrm{Co}$, and $\mathrm{Ni}$ atoms. Moving towards the middle of the $3 d$ transition series, for example for $\mathrm{Mn}$ and $\mathrm{Cr}$, a principle limitation of the sum rule approach becomes evident. The experimental spectroscopist observes that the $L_{3}$ and $L_{2}$-edges appear to "overlap", leading to systematic errors in the moment determination. The set of quantum numbers used in deriving the sum rules appears not to be the appropriate one [28]. Here again a relative sum rule analysis minimizes the errors, as the "edge overlap" takes place for both the "standard" and the "unknown". In such cases it is best to also perform theoretical calculations and not to only rely on empirical correction factors. Several codes are available to the experimentalist, allowing to perform calculations close to the edge. Even if those are not always fully satisfactory, at least they allow to obtain a degree of reliability and limit potential errors [25, 29-33].

It is possible to limit the uncertainties in magnetic moment determination, by repeating the same XMCD measurement and by varying the different control parameters, which the experiment provides. As an example, the XAS and XMCD data of Fig. 7 are for bulk $b c c$ Fe in thin film form, exhibiting high degree of crystal order and purity, which can then form practically a magnetic monodomain, once magnetized. In (a) one possibility to take a dichroic XMCD pair is shown. Here one keeps the magnetic state of the sample identical and reverts the light helicity $\left(\sigma^{+}\right.$ versus $\left.\sigma^{-}\right)$. In the case of the data shown here, this is obtained by means of reverting the phase of the elliptically polarizing undulator. One performs here the sum rule analysis. It is important to verify that the spectra coincide in the pre-edge and post-edge regions, photon energies where the dichroic asymmetry should be either zero (pre-edge) or practically negligible (high energies) as compared to the dichroic "white line" intensities. An empirical double step function is fitted. As highlighted 
fixed magnetisation direction reversed light helicity
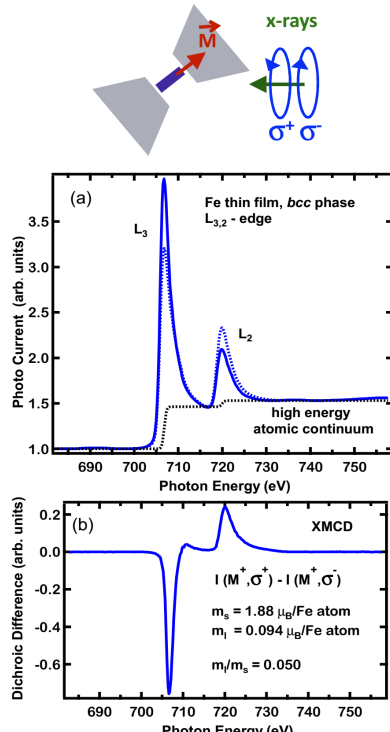

Energy (eV) fixed light helicity,
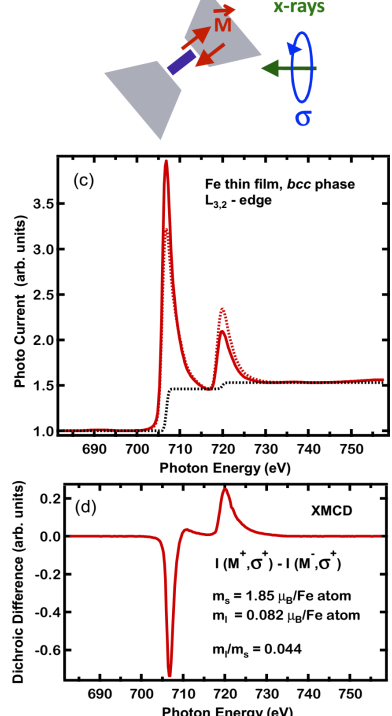
reversed magnetisation direction

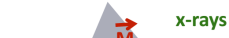

Fig. 7. We show XAS spectra (a, c) and their XMCD difference $(\mathrm{b}, \mathrm{d})$ at the $L_{3,2}$-edges of $\mathrm{Fe}$, for a metallic film of $b c c$ Fe. The figure illustrates two equivalent approaches to measure the XMCD effect. Both methods give the same result within the error. As illustrated in the schematic graphs above, the XMCD measurement can be done by applying two different experimental geometries: fixed magnetization direction and reversing the light helicity $(\mathrm{a}, \mathrm{b})$ or for a fixed light helicity and reversing the magnetization direction $(\mathrm{c}, \mathrm{d})$.

earlier, here the 1 to 2 step intensity for the $L_{3}$ and $L_{2^{-}}$ edges is not followed, one rather matches in an empirical manner the spectral shape. A number of $3 d$ holes of 3.8 is used for Fe. This number, in combination with the use of orbital polarization, gives good results in ground state calculations for the magnetic moments [34]. Using this step function, in combination with this number of ground state $3 d$ holes, a good agreement with previous experimental work in the transmission mode is obtained [35]. The integral areas are shown in the right axis in Fig. 8. One observes clearly the saturation of the summed spectral area versus photon energy at high photon energies above the $L_{2}$-edge, indicating that the integrated areas in this case have reached their final value. Still the $L_{2}$ "white line" has a long asymmetric tail at high energies, which indicates that some intensity is still to be recovered at high photon energies, pointing out any high energy cutoff as arbitrary. Here a relative analysis versus a "standard" would eliminate any systematic errors introduced by the finite energy bounds of the experiment.

In Fig. 8b the dichroic difference is also given, from where, following the sum rules, the orbital to spin moment ratio is obtained, neglecting the tensor term. This is justified as here we are dealing with a thick film of cubic symmetry where surface or other low coordination effects can be safely neglected. The integrated spectral area for the difference spectrum is also shown in the right scale.

The orbital to spin moment determination can be done by using the quantities $p$ and $q$ as shown in the Fig. 8b, according to the magneto optic sum rules neglecting the tensor term. Again, a very good agreement is obtained versus earlier work for $b c c$ Fe. Still any arbitrariness in reading these quantities in the right scale is eliminated in a relative analysis versus a "standard". Of importance is to observe as in Fig. 8a that the spectral areas contain no spurious contribution due an experimental artifact. If the dichroic pair spectra do not "match" in terms of spectral response of the beam line a spurious contribution may be obtained. This in term would be visible in particular outside the photon energies where there is dichroic contrast. For the integrated areas shown in Fig. 8b one would, for example, expect an increasing linear contribution. Here the dichroic pair spectra match well and the difference spectrum is flat. As a consequence the integral value also "saturates" at high photon energies. Finally, it is worthwhile to mention that as seen in the Fig. $8 \mathrm{~b}$ the inter-peak region presents a very specific type of dichroic response. In agreement to previous studies one observes that for $\mathrm{Fe}$ a small positive dichroic intensity is visible as referred to the negative $L_{3}$ dichroism. This small signal has been assigned earlier to transitions to $4 \mathrm{~s}$ final states [36]. Here the strength of the dipole matrix elements makes that the $4 s$ final states are seen less by a factor of about 50 and are therefore much smaller versus the $3 d$ ones. It influences the overall outcome of the magnetic moment analysis, and should be subtracted, increasing the degree of arbitrariness in the data treatment. Again a relative analysis may be the best solution keeping the same procedure for all spectra analyzed. This $4 s$ spectral region presents known systematics as one varies the atomic number along the elements of the $3 d$ transition series [36].

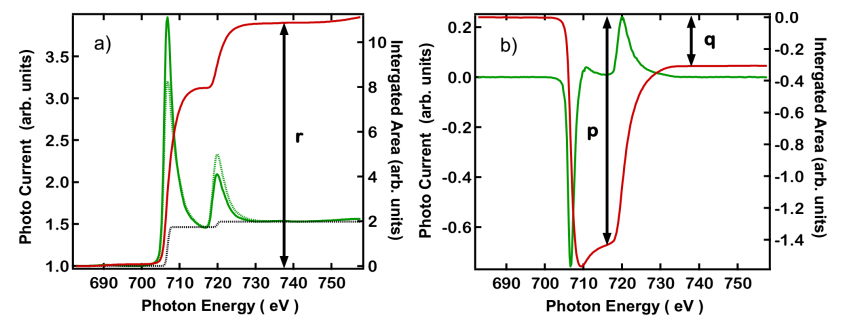

Fig. 8. We show the analysis of the dichroic areas for the $L_{3,2}$-edges of Fe to apply the magneto-optic XMCD sum rules. The half sum of the dichroic pair of the XAS spectra (a, left scale) and the integral of its area (right scale) are given in the left panel. The integrated dichroic area (b, left scale) is shown in the right panel. The $p, q$ and $r$ parameters, shown in the figures, are included in the sum rules equations. In panel (a) is also shown the atomic step function.

In Fig. 9, in situ XMCD spectra on Co thin films are shown, based on results of surface science experiments, to 
illustrate in a practical manner the lifting of the quenching of the orbital moment in a $3 d$ ferromagnet, an interesting physical effect. The same type of sum rule analysis, as for Fe in Fig. 7, is shown for two different Co films in Fig. 9. In Fig. 9a and b, one deals with an ultrathin film of fct Co grown and measured in situ in ultra high vacuum, on a clean and single crystalline $\mathrm{Cu}(100)$ surface. The surface cleanness was checked in situ by XAS at the $\mathrm{C}$ and $\mathrm{O} K$-edges, the surface order by means of in situ low energy electron diffraction (LEED), at all stages of the growth. $\mathrm{Ar}^{+}$sputter anneal cycles were used to clean and order the $\mathrm{Cu}$ surface.

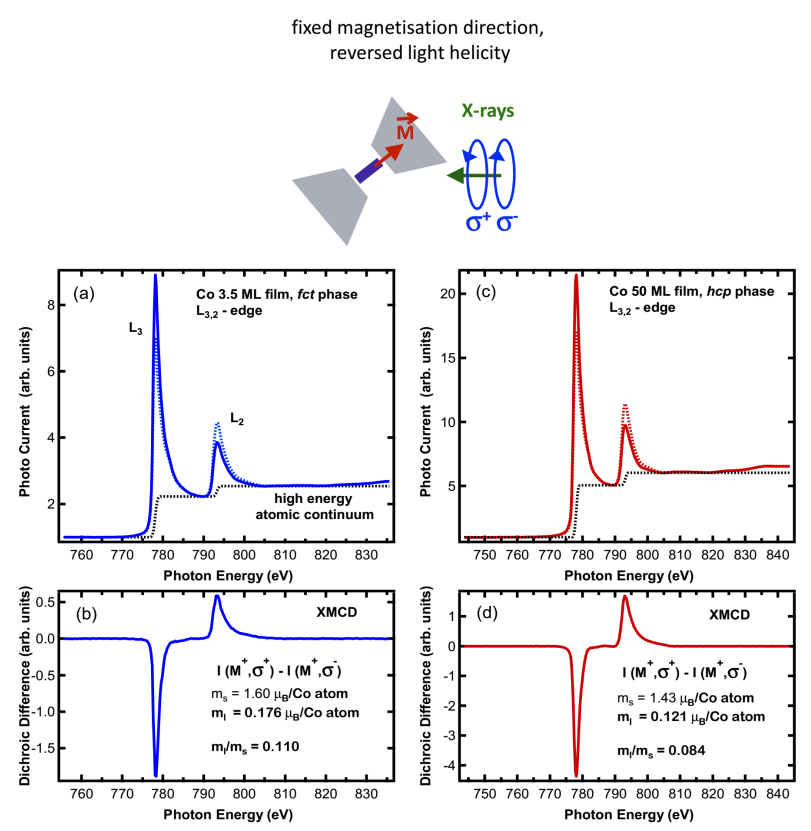

Fig. 9. We show XAS spectra (a, c) and the corresponding XMCD difference $(\mathrm{b}, \mathrm{d})$ at the $L_{3,2}$-edge of Co for a metallic 3.5 ML thin film of $f c t$ Co $(\mathrm{a}, \mathrm{b})$ and thicker, $50 \mathrm{ML}$, film of $h c p$ Co. The outcome of the sum rule analysis is included in $\mathrm{b}, \mathrm{d}$. These results illustrate in particular a strong increase of the orbital magnetic moment for the Co atoms of the surface layer of the Co film (see text).

The Co film is grown in situ by electron beam evaporation [37]. The experiments shown here were performed at the BL I1011 of MAX-lab. Here we want to illustrate, by the sum rule analysis, previous results in the literature. The thickness of the Co films can be determined via a comparison to previous sets of data in the literature, by means of the intensity of the Co atomic steps versus the $\mathrm{Cu}$ background intensity. The data are taken by measuring the photocurrent of the sample, or in "total electron yield" mode. The double step functions are shown in Fig. 9a and $\mathrm{c}$ and allow to determine the thickness precisely in conjunction with a quartz crystal microbalance calibration [13]. In Fig. 9c and d a much thicker Co film is grown on a $\mathrm{Cu}(111)$ single crystalline surface prepared in ultra high vacuum and characterized by means of XAS and LEED. For this film the Co adopts a structure like for the hcp bulk. Comparing the results of the sum rule analysis one observes that the 50 atomic layer thick $h c p$, bulk like, Co film exhibits spin and orbital moments in agreement to the literature for bulk Co values [35]. In contrast, the 3.5 atomic layer presents a enhancement of about $12 \%$ in the spin moment and about $50 \%$ for the orbital moment. Here the tensor term correction is neglected in the sum rules, a theoretical input would be needed, or angle dependent XMCD measurements versus a strong applied magnetic field, much stronger than the coercive field [38]. Here the data are taken along the in plane easy direction of the sample only, given the supplementary complication of the in situ growth. Ab initio theory, including the orbital polarization correction [39] predicts an enhancement of order of $100 \%$ for the orbital moment of the outermost surface layer facing the vacuum, as well as an enhancement of the spin moment of order of $10 \%$. The $50 \%$ enhancement of the Co atom orbital moment observed here, is in good agreement with the theory. Indeed, the inner Co layers close to the surface, do not experience such a strong enhancement for the orbital moment as they have the bulk number of first nearest neighbors [39]. The results given here are in good agreement with previous observations [37]. This lowering of local symmetry lifts the "quenching" of the orbital moment and has strong implications for the magnetic anisotropy of ultrathin films [18].

\subsection{Dichroism with linearly polarized light}

Despite the fact that most of the recent literature on dichroism effects focus on applications of XMCD, a spectroscopy based on circular X-rays, which is used to study ferromagnetic materials, X-ray dichroism is also possible on antiferromagnets. For an antiferromagnet, below the temperature where long range magnetic order occurs, the Neel temperature, adjacent spins are in an anti parallel arrangement. As the macroscopic net magnetic moment is zero for an antiferromagnet, no XMCD signal can be observed using circular X-ray light. This is a strong motivation in order to consider dichroism experiments on magnetic materials, which are based on a different experimental geometry, that would yield a non zero signal for atomic moments with an antiparallel alignment between nearest neighbor atoms. Therefore, in this part experiments based on XLMD are discussed.

Here a simple qualitative discussion is given, trying to avoid complicated symmetry arguments and mathematical formalism. In a simplified qualitative picture, thinking in terms of individual localized atomic magnetic moments, while the intensity of the XMCD signal is linear versus the strength of the atomic magnetic moment, and leads to a linear dependence upon the macroscopic magnetization, $M$, the XLMD intensity is proportional to the square of the atomic moment, and therefore is non zero for antiferromagnets. All the contributions of the individual atomic moments to the XLMD will sum up for either an antiferromagnetic arrangement, or ferromagnetic arrangement of the individual moments. 


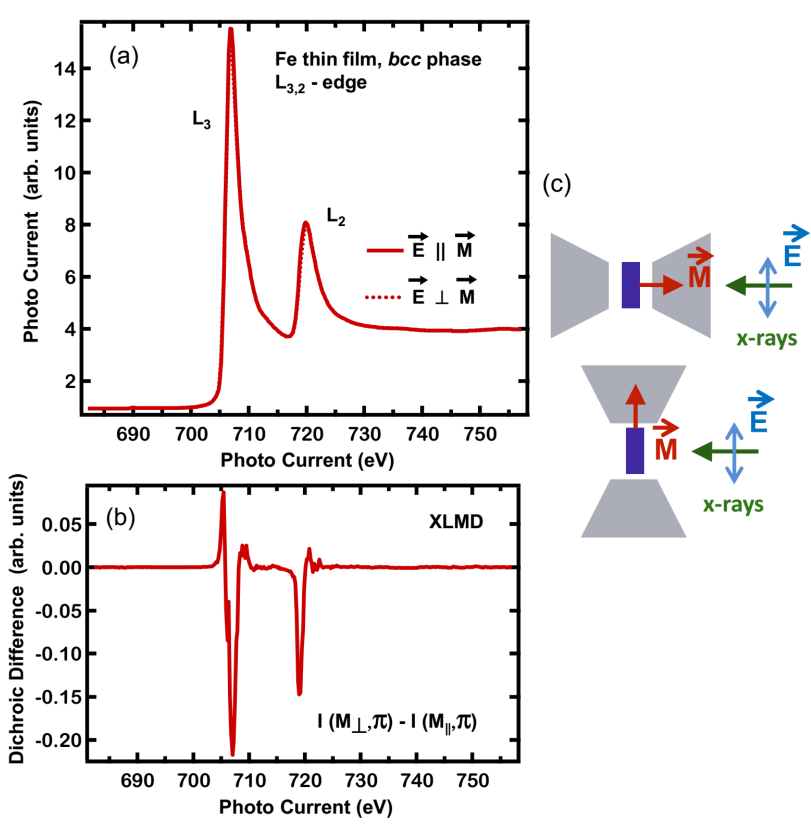

Fig. 10. We show XAS (a) and XLMD (b) spectra at the $L_{3,2}$-edges of $\mathrm{Fe}$ for a metallic film of bcc Fe. The XLMD measurements are done using linearly polarized light, the geometry of the measurement is shown schematically in panel c. For the XLMD measurement the angle of X-ray incidence is fixed, while the magnetization direction or the electric field direction is changed. In panel (c) we modify the magnetization direction of the sample by applying the magnetic field either in the surface plane or perpendicular to it.

In Fig. 10 XLMD data are shown on an Fe ferromagnetic thin film. The fact that both XMCD and XLMD data are measured on the same sample, allows to set an order of magnitude for the small XLMD effect. While the XMCD effect is of the order of $30 \%$ of the $L_{3}$ "white line" intensity, the XLMD effect is much smaller, of order $2 \%$ only. The strength of the XLMD effect appears therefore to be clearly well below $10 \%$ of the strength of the XMCD effect, considering the data of Fig. 7 and Fig. 10. This explains also why there is much less literature on the XLMD effect. However, for antiferromagnets it is the technique of choice to obtain dichroic contrast and for example, obtain photoelectron microscopy micrographs [16]. In particular XLMD provides the possibility to investigate the coupling between a ferromagnet and an antiferromagnet, at their common interface, as this signal is non zero in both cases [40]. In Fig. 10, a typical layout for XLMD on a ferromagnetic $b c c$ Fe thin film is also shown. The same geometry can be used for an antiferromagnet. Linear X-rays are used, and one compares the $\mathrm{X}$-ray absorption coefficient of the Fe film with the magnetization parallel and perpendicular to the electric field vector of the incoming radiation. In the geometry used in the figure, one keeps the angle of X-ray incidence constant, at normal X-ray incidence, and rotates the magnetization and/or the electric field vector by means of an appropriate X-ray source, such as an elliptically polariz- ing undulator. The reason is the small intensity of the XLMD effect, as one tries to minimize spurious experimental artifacts. Comparing data at various X-ray incidence angles, often leads to variable spectral baselines, which are then not easy to compare in order to extract the small XLMD difference, shown in Fig. 10. Once the XLMD difference is obtained for various elements one can quantitatively study the variation of the XLMD strength or intensity versus the value of the magnetic moment. One can indeed establish experimentally the $M^{2}$ dependence [41], and quantitatively also study with a higher degree of accuracy the strength of the XLMD versus the XMCD effect. An interesting development in this context is the existence of a sum rule, linking the XLMD signal with the orbital moment and the magneto crystalline anisotropy [42]. Given the weak strength of the XLMD signal only in very few cases a quantitative analysis is attempted in the literature [43].

\subsection{Dilute magnetic semiconductors}

The examples discussed so far did concern the XMCD measurements of thick films of transition metals, a fact which translates into strong dichroic signals. However, as mentioned in the introduction of the chapter, the XMCD technique is very sensitive and can detect even signals of materials called diluted magnetic semiconductors (DMS), and nanostructures. An example, which will be discussed below, relates to the $(\mathrm{Ga}, \mathrm{Fe}) \mathrm{N}$ film. For this sample the content of iron diluted in GaN is only $1 \%$. The Fe is diluted in both the matrix (substituting Ga atoms) as well as in iron-rich nanocrystals $\mathrm{FeN}_{x}$, where $\mathrm{x}<1$ [44].

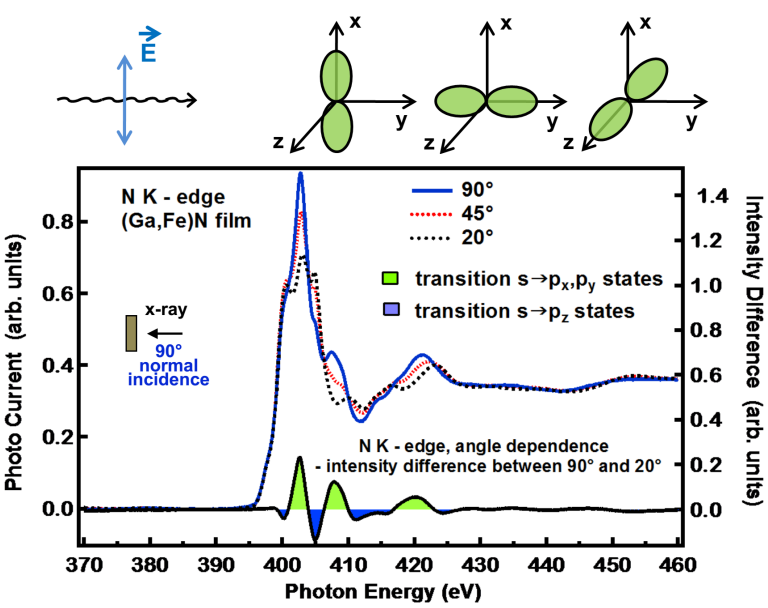

Fig. 11. We show the angle dependence of the X-ray absorption spectra at the $K$-edge of nitrogen in a $(\mathrm{Ga}, \mathrm{Fe}) \mathrm{N}$ thin film. The measurements are done using linearly polarized X-rays for the XAS spectra shown here. The spectra are measured for the X-ray incidence angle versus the sample surface at $90^{\circ}, 45^{\circ}$ and $20^{\circ}$. The strong angle dependence of the X-ray absorption is similar as in the case of non doped GaN [45].

First, we discuss the angular dependence of the absorption $K$-edge of N, illustrated in Fig. 11. Despite the 
presence of small amounts of Fe the $\mathrm{N} K$-edge spectra are very close to spectra of pure GaN [45]. Here one deals with the $\mathrm{N}(1 s)$ as the initial state and probes the $\mathrm{N}(2 p)$ final states. For the data of Fig. 11, linearly polarized $\mathrm{X}$-rays are used as illustrated in the figure. For this particular experiment, the electric field vector is oriented in the horizontal plane, and the sample polar angle is varied to modify the angle of X-ray incidence. At $90^{\circ}$ normal incidence, the electric field vector is within the surface plane, at $20^{\circ}$ grazing $\mathrm{X}$-ray incidence the electric field vector is $20^{\circ}$ off the surface normal. A strong angular dependence is observed in the X-ray absorption. This effect can be understood by a different population of the available $\mathrm{N}\left(2 p_{x}\right), \mathrm{N}\left(2 p_{y}\right)$ and $\mathrm{N}\left(2 p_{z}\right)$ final states, shown in Fig. 11. At normal incidence the $\mathrm{N}\left(2 p_{x}\right)$ and $\mathrm{N}\left(2 p_{y}\right)$ states are probed. One observes a stronger XAS intensity around $402 \mathrm{eV}$ incident photon energy, indicating the existence of final states of this symmetry. In contrast at this energy the $\mathrm{N}(2 p)$ final states posses much less $2 p_{z}$ character.

In Fig. 11 the difference spectrum allows to quantify this effect versus energy. The strong angle dependence can be observed when the GaN lattice possesses a high degree of crystalline order. As one here probes only the polar angle, it is sufficient for the specific GaN sample to be $c$-axis oriented. Also LEED spots were observed in this case, confirming this result in agreement to other observations for the specific sample [44]. This effect can be easily described mathematically for $2 p$ states in a localized picture of the electronic states, forming molecular orbitals in a molecule [46]. The $1 s$ initial state shows no angle dependence and the $2 p$ final states possess a "vector character" [46]. This leads for the measured dipole matrix element to a $(\cos \theta)^{2}$ angle dependence the angle $\theta$ being the angle between the direction of maximum orbital density of the final $2 p$ state and the electric field vector. This "search light" effect in the XAS intensity of $K$-edges is reminiscent of the angle dependence of the $K$-edge EXAFS intensity for single crystalline materials.

XMCD measurements were made both for the Fe $L$ edge (Fig. 12a and b), and the N K-edge (Fig. 12c and d). In both cases, measurements were made at $300 \mathrm{~K}$ in a magnetic field about 800 Oe (in the plane of the sample). The exact energy position of the $L$-edge, the spectral broadening and intensity, carry information on the electronic configuration of the specific iron atoms. The energy and intensity of the Fe $L$-edges have been calibrated by comparison with a "standard" sample of Fe $b c c$ measured in situ. The observed broadening of the $L_{3}$ "white line" for ( $\mathrm{Ga}, \mathrm{Fe}) \mathrm{N}$ sample compared to the standard Fe, indicates the presence of iron in several different environments and local crystallographic phases. Using the sum rules the spin and orbital magnetic moment per iron atom were obtained (values are given in Fig. 12d).

As mentioned in the beginning of the chapter, the $\mathrm{XMCD}$ technique is an element specific technique, while it also allows to examine the contribution of the constituent elements to the total magnetic moment of the
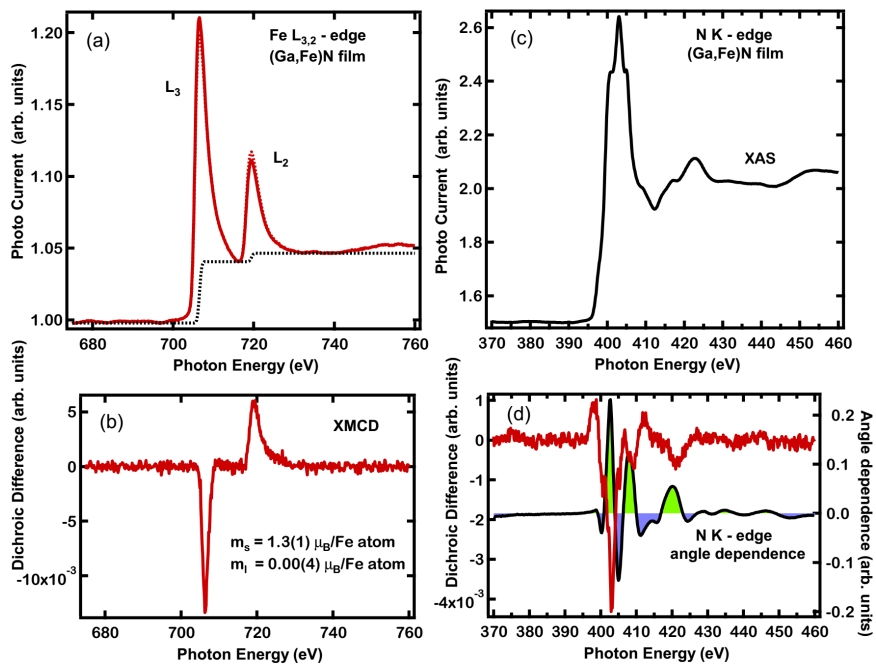

Fig. 12. We show XAS and XMCD spectra at the $L_{3,2^{-}}$ edges of $\mathrm{Fe}(\mathrm{a}, \mathrm{b})$ and at the $K$-edge $(\mathrm{c}, \mathrm{d})$ of $\mathrm{N}$ in a $(\mathrm{Ga}, \mathrm{Fe}) \mathrm{N}$ thin film. The measurements were done under an applied field of 800 Oe. The spectra at the Fe $L_{3,2}$ absorption edges were taken at $45^{\circ} \mathrm{X}$-ray incidence, the ones at the N $K$-edge at normal X-ray incidence. An $\mathrm{XMCD}$ difference is clearly seen for both elements.

sample. As it turns out, in the case of $(\mathrm{Ga}, \mathrm{Fe}) \mathrm{N}$, besides the dominant contribution to the magnetic signal carried by Fe ions, part of the spin polarization is also carried by nitrogen atoms. In the case of the $\mathrm{N} K$-edge we start from initial states of $s$ symmetry and we probe $\mathrm{N}(2 p)$ final states, given the dipole selection rule. Because the initial states of $s$ symmetry have no finite orbital moment and the associated spin-orbit energy is zero, in $K$-edge XMCD measurements we loose in XMCD sensitivity, versus XMCD detected using $p$ symmetry initial states. Working with a $K$-edge leads therefore to a greatly reduced sensitivity to detect the orbital magnetic moment of the photoexcited atom. Moreover, the $p$ symmetry states are much less spin polarized than $3 d$ states, so the XMCD effect observed at a $K$-edge is several orders of magnitude weaker versus the one observed at $L_{3,2}$-edges. It does not exceed the tenth part of the percentage of the atomic cross section, whereas in the case of $L$-edges this effect can be even higher than $60 \%$ in some cases.

However, in the case of the $(\mathrm{Ga}, \mathrm{Fe}) \mathrm{N}$ film, under the external magnetic field we observe a clear dichroic signal coming from the nitrogen atoms.

A strong dichroic signal is observed in the direction of the normal to the surface, as shown in Fig. 12d. Previously, this effect was also observed for the $K$-edge of nitrogen in the GdN [47]. This phenomenon is a direct confirmation of electron transfer and spin polarization between the $\mathrm{Fe}$ and the neighboring ions through spindependent hybridization between the $\mathrm{N}(2 p)$ with $\mathrm{Fe}(3 d)$ and $\mathrm{Fe}(4 s)$ states. In particular, a strong XMCD effect is visible in the energy of $397 \mathrm{eV}$, just before the absorption 
edge, which corresponds to the forbidden energy range within the band gap. This signal is comparable with the results published for $\mathrm{GdN}$ and it is about $0.5 \%$ of the high energy continuum of the absorption spectrum $[47,48]$.
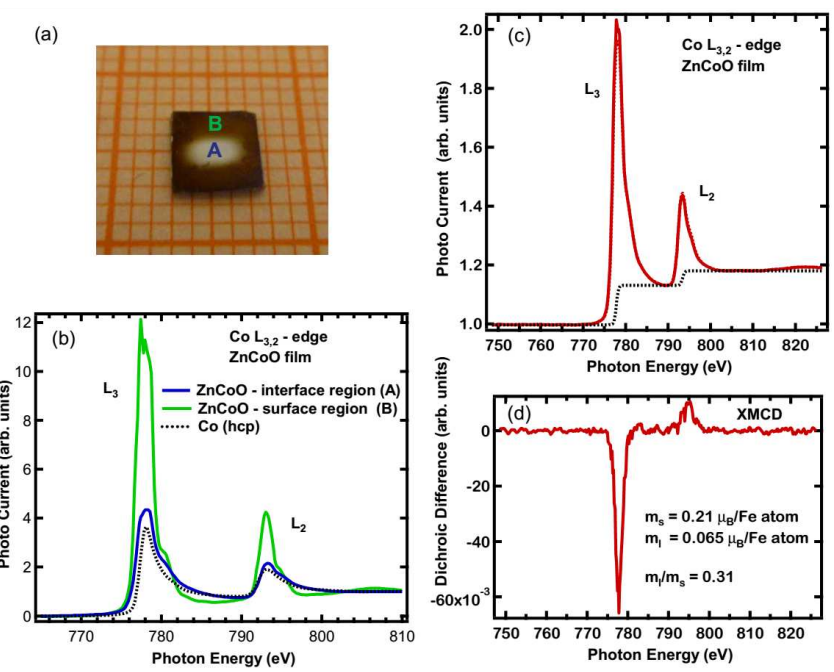

Fig. 13. We show XAS and XMCD results obtained at the Co $L_{3,2}$-edges in a $(\mathrm{Zn}, \mathrm{Co}) \mathrm{O}$ thin film. The measurements were done both at the interface and inside the crater obtained by $\mathrm{Ar}^{+}$ion sputtering (A) and at the sample surface (B) as marked in panel (a), which shows a photograph of the sample. Panel (b) shows XAS spectra obtained for both regions (A and B) as well as a metallic Co film, using linearly polarized light. The metallic Co film is used as a reference (a "standard") to characterize the "white line" intensity. The spectra were normalized on a per atom basis. In panel (c) are shown XAS spectra measured using circularly polarized light under an applied magnetic field of 350 Oe at an X-ray incidence angle of $40^{\circ}$ versus the sample surface. The corresponding XMCD difference is shown in panel (d).

The XAS and XMCD spectra allow to distinguish the electronic state of an element, as it is illustrated in the next example concerning $(\mathrm{Zn}, \mathrm{Co}) \mathrm{O}$ films. This sample was probed separately both in the surface and the interface region with a Si substrate, locations $\mathrm{A}$ and $\mathrm{B}$, respectively, as marked in Fig. 13a. The interface region was probed within the crater previously etched down to the layer/substrate interface by $\mathrm{Ar}^{+}$ion sputtering. The size of the beam spot was reduced to match the size of the crater, to about $0.8 \times 1.8 \mathrm{~mm}^{2}$. The content of Co in the "bulk" part of the layer is $6 \%$ on average, calculated by using the tabulated atomic cross sections for XAS. The distribution of $\mathrm{Co}$ is not homogeneous within the layer and the highest amount of Co is about $14 \%$.

As presented in Fig. 13b, we find sizable differences in the XAS fine structure of the Co "white lines" for the surface and the interface regions, when measured using linearly polarized light. The Co $L$-edge obtained at the surface region shows a typical multiplet shape, reported previously for $(\mathrm{Zn}, \mathrm{Co}) \mathrm{O}$. A different chemical composition and electronic structure are observed within the crater.
To compare the two results the spectra are normalized to the high energy continuum. In particular, we observe, that the interpeak continuum of final states (of $s$ symmetry) for the Co spectra is stronger in the crater. Since the magnitude of the final state continuum is a direct probe of the degree of metallic character, we conclude that Co may form either a continuous ultrathin film or a layer of nanocrystals at the interface. For comparison in Fig. 13b is shown also the XAS spectrum of metallic Co. At the same time, the shape of the XAS spectra indicates that in the interface region metallic $\mathrm{Co}$ is the dominant phase, but not the only one, as we detect also a contribution specific to $\mathrm{CoO}$. More quantitatively, the area under the $\mathrm{Co} L_{3}$ line (Fig. 13b) corresponding to the surface and interface region is, respectively, 2.5 and 1.25 times larger than expected for metallic Co. This observation shows, that while Co is mostly metallic at the interface, still about $17 \%$ of Co atoms are in a $2^{+}$state. At the same time, a certain amount of $\mathrm{Zn}$ has also been found at the interface, showing that metallic Co coexists with $(\mathrm{Zn}, \mathrm{Co}) \mathrm{O}$.

The difference in the electronic state of the Co atoms in the two probed regions is reflected also in their magnetic properties. The XMCD measurements were done at room temperature with a magnetic field of 350 Oe applied along the film plane. As shown in Fig. 13d, an XMCD dichroic signal is seen in the interface region, but not in the surface region. The dichroic effect is much stronger at the $L_{3}$ versus the $L_{2}$-edge, an observation which indicates a high orbital moment, much stronger than in case of a bulk cobalt sample (the values are shown in Fig. 13d). The $m_{l} / m_{s}$ ratio obtained by applying the magneto-optical sum rules for the $(\mathrm{Zn}, \mathrm{Co}) \mathrm{O}$ film is 0.31 , when the typical value for metallic Co is 0.08. Such a result is possible when Co atoms form small inclusions or nanocrystals [49].

\section{PEEM as a tool for XMCD and XLMD studies with lateral resolution}

With the unique properties of the radiation produced by third-generation synchrotron facilities (high intensity and brilliance, and a wide spectral range and the possibility of the light polarization), there has been great progress in the development of spectro-microscopic techniques, which combine the most important spectroscopic techniques with spatial resolution. A microscopy with a wide range of applications to surface studies, interfaces, thin films and nanostructures, is photoemission electron microscopy PEEM (photoemission electron microscopy). A major advantage of this technique, based on $\mathrm{X}$-rays (X-PEEM), is element specificity, which in combination with the spatial resolution, allows to image the distribution of a given element in the material. PEEM microscopy is based on several widely used spectroscopic techniques, based on the detection of electrons emitted from atomic core levels (XPS, UPS) and on the measurement of secondary electrons (allowing for XAS, XMCD, XLMD), often combined with low-energy electron microscopy (LEEM). 


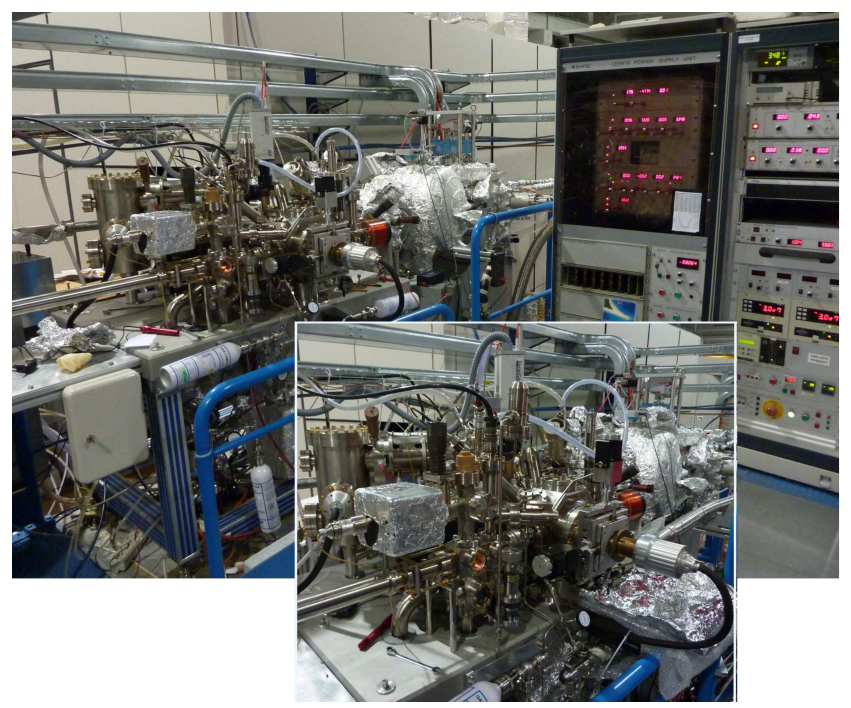

Fig. 14. We show photographs of the PEEM/XMCDPEEM microscope operating at the "Nanospectroscopy" beam line of the Elettra synchrotron facility in Trieste (Italy). The X-rays are coming from the left side in the picture. Part of the instrumentation is protected behind shields to increase the electric insulation, as the microscope operates at a high electrostatic voltage (see text). The sample is inserted first in a load lock system, then into a preparation chamber where it can be sputtered and annealed, before being inserted into the main microscope chamber for the PEEM measurements.

The PEEM technique combines the photoelectric effect with electron microscopy, using monochromatic X-rays, which leads to the emission of electrons. The acronym PEEM is a bit misleading and is given only for historical reasons, because PEEM microscopy techniques can be divided into two categories: the first group requires an energy filter and is based on the detection of electrons with an energy characteristic for the material (XPS, UPS), the second group uses the emission of secondary or Auger electrons with an energy characteristic for the material (XAS, XMCD, XLMD).

The spatial resolution is provided by electron optics, it depends on the size of the aperture and the operating voltage, and even the photon flux density [50]. The spatial resolution is limited by spherical aberration, chromatic aberration and diffraction. To some extent, the role of the chromatic aberration corrector, caused by the errors in the focusing of the electrons with different kinetic energies, is fulfilled by the aperture acting as a filter of high energy electrons. In this case, one can achieve a spatial resolution of $20 \mathrm{~nm}$. In modern PEEM microscopes, the aberration is further minimized through the use of an energy filter and an optical aberration corrector, which allows to achieve the resolving power of a few nanometers.

Imaging is done by the measurement of the photoelectrons and (mostly) secondary electrons, through the use of the assembly of electrostatic and/or magnetic lenses. Emitted electrons are accelerated by a high potential ap- plied between the sample and the first lens, called the objective lens (approximately $20 \mathrm{kV}$ ). So the sample is part of the optical system, as the cathode. The objective lens forms a magnified image of the electron current of the sample, which is further magnified by one or several lenses. The final image is obtained using a multielement detector (such as a multi-channel plate) and a fluorescent screen coated with phosphor, where the visible light emitted from the detector is recorded by a CCD camera.

In this subsection we do not want to fully discuss PEEM spectro-microscopy, we will focus only on that part of the PEEM applications which deal with magnetic microscopy, based on the XMCD and XLMD effects, which are the main subject of this article. More information one can find in the literature [16, 51]. This microscopy mode is based on absorption spectroscopy with spatial resolution, in which we detect the secondary electrons as a function of photon energy. With this technique we do not discriminate the electrons emitted from the sample in terms of their energy, as it takes place in photoemission spectroscopy. We measure all the emitted electrons, and the signal we use as a measure of the absorption of the material for a given photon energy. In this way, we obtain a map of the X-ray absorption of the material, which is proportional to the electron emission from the sample. The physical basis for the formation of magnetic contrast is the same as in the case of XMCD and XLMD effects discussed in the section 3.3.

The results of XMCD-PEEM, which we present below as an example of an application of the microscopy with magnetic contrast, were taken at the "Nanospectroscopy" beam line in the synchrotron laboratory Elettra (Trieste, Italy) (Fig. 14). The high spatial resolving power requires a high photon flux density in the sample area which will be imaged by the microscope. The "Nanospectroscopy" beam line is equipped with two undulators, which provide a flux of the order of $2 \cdot 10^{13}$ photons $/ \mathrm{sec}$ and linear polarization (horizontal and vertical) as well as elliptical one. The available energy range is $50-1000 \mathrm{eV}$. The size of the beamspot on the sample is 20 microns by $2-5$ microns. The beam line is equipped with a microscope from the Elmitec company. This system combines LEEM electron microscopy with PEEM (especially XMCD-PEEM and XLMD-PEEM). The laterally resolving power of the $\mathrm{X}$ PEEM microscopy is $40 \mathrm{~nm}$, in the XMCD-PEEM mode it is $50 \mathrm{~nm}$ and in the LEEM mode it is $15 \mathrm{~nm}$. In the LEEM mode the surface topography of the material is imaged, while in the XMCD-PEEM mode the in plane magnetic contrast is obtained of the same sample area. These techniques, together with the XAS, XMCD and XLMD measurements without spatial resolution, allow to obtain the complete picture of the magnetic properties of the studied material, with the determination of magnetic moments, the magnetic phases and their distribution with the determination of the contribution of each element.

As an example, Fig. 15 presents the PEEM results obtained for a $(\mathrm{Ga}, \mathrm{Fe}) \mathrm{N}$ film. Micrographs were measured 
(a) XAS Fe $L_{3}$ FOV $=1 \times 1 \mu \mathrm{m}$

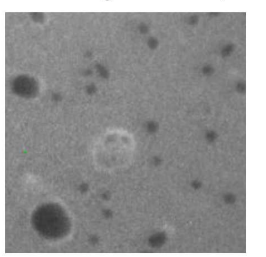

(b) $X M C D$ Fe $L_{3}$ FOV= $1 \times 1 \mu \mathrm{m}$

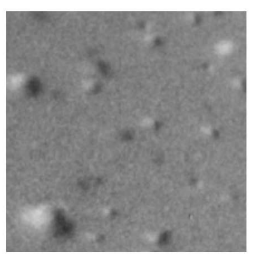

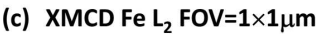

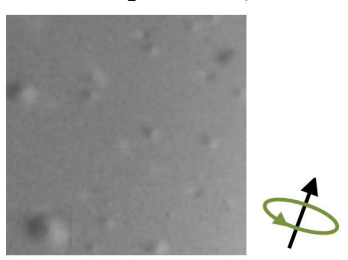

(e)

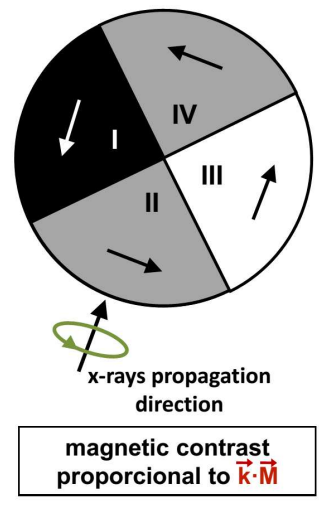

Fig. 15. We show PEEM micrographs measured at the "Nanospectroscopy" beam line at Elettra for a nanostructured FeN/GaN thin film. The field of view (FOV) is given for each panel. The microscope operates at grazing X-ray incidence so that only the in plane component of the magnetization is seen. The micrograph in panel (a) was obtained in the XAS mode, it allows to visualize the Fe-rich nanocrystals at the $(\mathrm{Ga}, \mathrm{Fe}) \mathrm{N}$ surface, seen as islands of darker contrast. The XAS spectra shown in panel (d) prove that almost all the Fe is distributed within the nanocrystals. The micrographs in panels (b) and (c) show the XMCD signal at the $L_{3}(\mathrm{~b})$ and $L_{2}$ (c) edges. The contrast is magnetic as it reverts between the two panels, an important experimental check. In the case of the larger nanocrystals, the characteristic magnetic contrast of a magnetic "vortex" is seen, which is schematically shown in panel (e). The arrows represent the average spin direction in this panel.

for the $L_{3}$ absorption edge of iron, the area visible in the figures is $1 \mu \mathrm{m}^{2}$. The first one (a) is obtained for the $L_{3}$-edge, using linearly polarized X-rays, and shows the spatial distribution of iron. The selected sample is characterized by Fe-rich nanocrystals of various sizes from $40 \mathrm{~nm}$ to $150 \mathrm{~nm}$. The dark contrast on the micrograph (a) is due to the Fe-rich nanocrystals, but a very small amount of iron is also embedded in the GaN matrix, a fact which is confirmed by the absorption spectra obtained from the area of a single nanocrystal and the doped GaN matrix (d). The spectra obtained with PEEM microscopy are of not as good quality as measured by XAS/XMCD without spatial resolution. In the latter case we get a much better signal to noise ratio, and therefore the information derived from them is exhibiting a much smaller error. This is one of the reasons why one should use both techniques, which are complementary to each other.
Performing XMCD-PEEM measurements for the same sample area, one can explore the magnetic properties and domain structure of individual nanocrystals. For this reason the measurements for both Fe edges, $L_{3}$ and $L_{2}$, were performed and their magnetic contrast is presented respectively in (b) and (c) micrographs, which are the result of subtracting two absorption images obtained for the same area of the surface using the left and right handed helicity of circularly polarized light. Measurements were performed at room temperature in the magnetic virgin state. Because of the opposite sign of the dichroic difference at $L_{3}$ and $L_{2}$-edges, also black and white contrast on the micrographs (b) and (c) should be inverted, what confirms the magnetic character. Due to the geometry of the measurement, in which $\mathrm{X}$-rays are incident on the surface of the material at $16^{\circ}$ grazing incidence, the contrast is the result of the magnetization component in the plane of the sample. All nanocrystals, regardless of their size, are magnetic, while the matrix in which they are embedded does not show dichroic contrast. Some of the smallest nanocrystals are single domain as their whole area is white or black, the contrast is reversed when the energy of the photons is tuned from the $L_{3}$ to the $L_{2}$-edge. Other nanocrystals have a domain structure in the form of a "vortex". This structure arises when the formation of a ferromagnetic domain wall becomes a disadvantage in terms of magnetostatic energy. For this type of structures, as the name suggests, the direction of the spins is rotating around the "vortex" core. In the PEEM micrographs the XMCD contrast of the "vortex" structures is visible as white and black areas, inverted for the $L_{3}$ (b) and $L_{2}$ (c) edges, whose total area is smaller than the size of the nanocrystal (a). To understand this picture, one should remember the principle of the formation of magnetic contrast for different configurations of measurement. The XMCD signal is proportional to $\boldsymbol{k} \cdot \boldsymbol{M}$, where $\boldsymbol{k}$ is the wave vector direction and $M$ is the magnetization of the sample. Therefore, the XMCD signal will be detected only for the configuration in which the projection of magnetization vector is parallel to the direction of the $\boldsymbol{k}$ vector. If the exciting radiation is incident on the sample at a small angle to the surface, the measured magnetization component will be mainly in the plane of the surface plane of the material. Therefore, the structure of the magnetic "vortex" can be schematically divided into four parts (in fact there is no domain wall), as illustrated in the Fig. 15e. The arrows marked in the middle of each part represent the resultant direction of spin. The direction of incidence of the circularly polarized X-rays is indicated by the arrow in the figure (c) and (e). On the micrographs showing the XMCD signal, the magnetically visible part of the structure will be the one in which the projection of the magnetization direction is parallel to the $\boldsymbol{k}$ vector, these are parts I and III in the Fig. 15e, and the color of them will be inverted as their magnetization direction is opposite. Parts II and IV do not give magnetic contrast, since the $\boldsymbol{k}$ and $\boldsymbol{M}$ vectors in both cases are perpendicular. 
As mentioned above, the XAS/XMCD/XLMD techniques without spatial resolution and in combination with microscopy are complementary and allow for a better understanding of the magnetic properties and its origin in the material. PEEM microscopy can examine the spatial distribution of the individual elements and the distribution of the magnetic signal, but also has limitations that can be avoided by doing the same measurements without spatial resolution. In the case of PEEM spectro-microscopy one can not apply a strong magnetic field, as it would influence the track of the detected electrons and the magnetic and electrostatic elements of the microscope optics. The temperature range of measurements is also strongly limited, in contrast to spectroscopies where the sample can be cooled both with liquid nitrogen and helium (above temperature of $30 \mathrm{~K}$ ). Some limitations may also arise by charging effects of the sample. In the case of low conductive materials there is a greater chance of a successful experiment using spectroscopy rather than spectro-microscopy, because in the latter case the strong photon flux is focused on a very small area of the sample, of the order of micrometers.

\section{Summary and outlook}

In the present overview article, some examples are given from the study of magnetic materials, using X-ray absorption spectroscopy in a synchrotron radiation laboratory. The element specificity of X-ray absorption spectroscopy allows to disentangle the magnetic moment in its components in the case of alloys or multicomponent systems. It is also possible to disentangle the contribution to the magnetic moment of the various electronic levels by the careful choice of the absorption edges studied, using the dipole selection rules of electronic transitions. The use of an elliptically polarizing undulator as an X-ray source of variable polarization presents advantages as no beam line components are needed to alter the polarization state of the X-ray beam, allowing for a high degree of accuracy in magnetic moment determination.

It is concluded that X-ray absorption dichroism using both circular and linear X-rays can fully characterize the magnetic ground state of both metallic and semiconducting magnetic materials. Using X-ray absorption allows to characterize dilute magnetic materials such as ultra thin films on surfaces grown in situ or dilute magnetic semiconductors. An advantage of the dichroic spectroscopies presented here is the determination of the orbital moment as well as the magnetic moment determination for anti-ferromagnetic and ferrimagnetic materials.

In the future we may still see some improvements of the use of X-ray absorption in spectro-microscopy applications with the appearance of aberration corrected PEEM instruments. We already can image the magnetic domain structure of single magnetic nanocrystals in present undulator based beam lines, as we show in this article. In Poland the Solaris light source will soon start operation. The present article provides an overview of the use of X-ray absorption for dichroism applications in the area of magnetism with key references, to serve as a general reference in particular for the new users interested in magnetism applications using synchrotron radiation and X-ray absorption in Poland.

\section{Acknowledgments}

We acknowledge the support of the Polish National Science Centre under Grant No. 2011/03/D/ST3/02654. We acknowledge also the support from the Baltic Science Link project coordinated by the Swedish Research Council, VR, and the Coordinated Access to Lightsources to Promote Standards and Optimization (CALIPSO) Transnational Access Program. I would like to thank D. Arvanitis from Uppsala University for several years of valuable collaboration. I thank T. Dietl from the IF PAN for the opportunity to collaborate and carry out research using the techniques described in this article. Thank you J.J. de Miguel from the University Autonoma the Madrid for your valuable collaboration all these years. Thank you M.A. Niño Orti from IMDEA and A. Locatelli from the Elettra synchrotron laboratory for valuable discussions on PEEM microscopy. I also acknowledge A. Bonanni from the Kepler University in Linz and M. Godlewski from the IF PAN for interesting samples and helpful discussions. I want to thank the Director of MAX-lab N. Mårtenson for the opportunity to work in this synchrotron laboratory and be part of the I1011 buildup team. I thank MAX-lab staff, E. Wallén for support with the undulator calibration, B.N. Jensen for his support in tuning the I1011 beam line and G. Öhrwall for join work on the I1011 beam line.

\section{References}

[1] B. Jones, in: The life and letters of Faraday, Ser. Cambridge Library Collection, Cambridge University Press, Cambridge 2010, Vol. 2, p. 194.

[2] H.S. Bennet, E.A. Stern, Phys. Rev. B 137, A448 (1965).

[3] J.L. Erskine, E.A. Stern, Phys. Rev. B 12, 5016 (1975).

[4] B.T. Thole, G. van der Laan, G.A. Sawatzky, Phys. Rev. Lett. 55, 2086 (1985).

[5] G. van der Laan, B.T. Thole, G.A. Sawatzky, J.B. Goekoop, J.C. Fuggle, J.M. Esteva, R. Karnatak, J.P. Remeika, H.A. Dabkowska, Phys. Rev. B 34, 6529 (1986).

[6] G. Schütz, W. Wagner, W. Wilhelm, P. Kienle, R. Zeller, R. Frahm, G. Materlik, Phys. Rev. Lett. 58, 737 (1987).

[7] G. Schütz, M. Knülle, R. Wienke, W. Wilhelm, W. Wagner, P. Kienle, R. Frahm, Z. Phys. B 73, 67 (1988).

[8] G. Schütz, R. Frahm, P. Mautner, R. Wienke, W. Wagner, W. Wilhelm, P. Kienle, Phys. Rev. Lett. 62, 2620 (1989).

[9] C.T. Chen, F. Sette, Y. Ma, S. Modesti, Phys. Rev. B 42, 7262 (1990).

[10] P. Carra, B. T. Thole, M. Altarelli, X. Wang, Phys. Rev. Lett. 70, 694 (1993). 
[11] B.T. Thole, P. Carra, F. Sette, G. van der Laan, Phys. Rev. Lett. 68, 1943 (1992).

[12] E. Hecht, Optics, Addison Wesley, 1987.

[13] D. Arvanitis, M. Tischer, J.H. Dunn, F. May, N. Martensson, K. Baberschke, in: Experimental determination of orbital, spin moments from MCXD on $3 d$ metal overlayers, Spin-Orbit-Influenced Spectroscopies of Magnetic Solids, ser. Lecture Notes in Physics, Vol. 466, Eds.: H. Ebert, G. Schütz, Springer, Berlin 1996, p. 145.

[14] hreffcxro.lbl.govwww.cxro.lbl.gov.

[15] J.H. Dunn, D. Arvanitis, N. Martensson, M. Tischer, F. May, M. Russo, K. Baberschke, J. Phys.: Condens. Matter 7, 1111 (1995).

[16] J. Stöhr, H.C. Siegmann, in: Magnetism from fundamentals to nanoscale dynamics, ser. Springer Series in Solid-State Sciences, Vol. 152, Springer, Berlin 2006.

[17] J. Stöhr, H.A. Padmore, S. Anders, T. Stammler, M.R. Scheinfein, Surf. Rev. Lett. 5, 1297 (1998).

[18] P. Bruno, Phys. Rev. B 39, 865 (1989).

[19] D. Weller, J. Stöhr, R. Nakajima, A. Carl, M.G. Samant, C. Chappert, R. Mégy, P. Beauvillain, P. Veillet, G. Held, Phys. Rev. Lett. 75, 3752 (1995).

[20] A. Aspelmeier, M. Tischer, M. Farle, M. Russo, K. Baberschke, D. Arvanitis, J. Magn. Magn. Matter. 146, 256 (1995).

[21] C. Stamm, T. Kachel, N. Pontius, R. Mitzner, T. Quast, K. Holldack, S. Khan, C. Lupulescu, E.F. Aziz, M. Wietstruk, H.A. Dürr, W. Eberhardt, Nat. Mater. 6, 740 (2007).

[22] J. Stöhr, J. Magn. Magn. Matter. 200, 470 (1999).

[23] J.-M. Mariot, C. Brouder, in: Spectroscopy, Magnetism: An Introduction, Magnetism, Synchrotron Radiation, ser. Lecture Notes in Physics, Vol. 565, Eds.: E. Beaurepaire, F. Scheurer, G. Krill, J.-P. Kappler, Springer, Berlin 2001, p. 24.

[24] P. Söderlind, O. Eriksson, B. Johansson, R.C. Albers, A. M. Boring, Phys. Rev. B 45, 12911 (1992).

[25] A.I. Nesvizhskii, A.L. Ankudinov, J.J. Rehr, Phys. Rev. B 63, 094412 (2001).

[26] M. Altarelli, Il Nuovo Cimento 20D, 1067 (1998).

[27] C. Piamonteze, P. Miedema, F.M.F. de Groot, Phys. Rev. B 80, 184410 (2009).

[28] H. Wende, A. Scherz, F. Wilhelm, K. Baberschke, J. Phys.: Condens. Matter 15, S547 (2003).

[29] M.P. Prange, J.J. Rehr, G. Rivas, J.J. Kas, J.W. Lawson, Phys. Rev. B 80, 155110 (2009).

[30] J. Vinson, J.J. Rehr, J.J. Kas, E.L. Shirley, Phys. Rev. B 83, 115106 (2011).

[31] C.R. Natoli, M. Benfatto, C. Brouder, M.F.R. Lopez, D.L. Foulis, Phys. Rev. B 42, 1944 (1990).

[32] K. Hatada, K. Hayakawa, J. Chaboy, C.R. Natoli, J. Phys.: Conference Series 190, 012010 (2009).
[33] D.D. Vvedensky, D.K. Saldin, J.B. Pendry, Computer Phys. Comm. 40, 421 (1986).

[34] O. Eriksson, A.M. Boring, R.C. Albers, G.W. Fernando, B.R. Cooper, Phys. Rev. B 45, 2868 (1992).

[35] C.T. Chen, Y.U. Idzerda, H.J. Lin, N.V. Smith, G. Meigs, E. Chaban, G.H. Ho, E. Pellegrin, F. Sette, Phys. Rev. Lett. 75, 152 (1995).

[36] W.L. O'Brien, B.P. Tonner, Phys. Rev. B 50, 672 (1994).

[37] M. Tischer, O. Hjortstam, D. Arvanitis, J.H. Dunn, F. May, K. Baberschke, J. Trygg, J.M. Wills, B. Johansson, O. Eriksson, Phys. Rev. Lett. 75, 1602 (1995).

[38] J. Stöhr, H. König, Phys. Rev. Lett. 75, 3748 (1995).

[39] O. Hjortstam, J. Trygg, J.M. Wills, B. Johansson, O. Eriksson, Phys. Rev. B 53, 9204 (1996).

[40] A. Scholl, H. Ohldag, F. Nolting, S. Anders, J. Stöhr, in: Study of ferromagnet antiferromagnet interfaces using x-ray PEEM, Magnetic Microscopy of Nanostructures, ser. NanoScience, Technology, Eds.: $\mathrm{H}$ Hopster, H.P. Oepen, Springer, Berlin 2005, p. 29.

[41] M. M. Schwickert, G.Y. Guo, M.A. Tomaz, W.L. O'Brien, G.R. Harp, Phys. Rev. B 58, R4289 (1998).

[42] G. van der Laan, Phys. Rev. Lett. 82, 640 (1999).

[43] S.S. Dhesi, G. van der Laan, E. Dudzik, A.B. Shick, Phys. Rev. Lett. 87, 067201-1 (2001).

[44] A. Navarro-Quezada, W. Stefanowicz, T. Li, B. Faina, M. Rovezzi, R.T. Lechner, T. Devillers, G. Bauer, M Sawicki, T. Dietl, A. Bonanni, Phys. Rev. B 81, 205206 (2010).

[45] M. Katsikini, E.C. Paloura, M. Fieber-Erdmann, E. Holub-Krappe, D. Korakakis, T.D. Moustakas, J. Electron Spectrosc. 101-103, 695 (1999).

[46] J. Stöhr, in: NEXAFS spectroscopy, ser. Springer Series in Surface Sciences, Vol. 25, Springer, Berlin 1992.

[47] V.N. Antonov, B.N. Harmon, A.N. Yaresko, A.P. Shpak, Phys. Rev. B 75, 184422 (2007).

[48] I.A. Kowalik, A. Persson, M.A. Ninõ, A. NavarroQuezada, B. Faina, A. Bonanni, T. Dietl, D. Arvanitis, Phys. Rev. B 85, 184411 (2012).

[49] M. Sawicki, E. Guziewicz, M.I. Łukasiewicz, O. Proselkov, I.A. Kowalik, W. Lisowski, P. Dluzewski, A. Wittlin, M. Jaworski, A. Wolska, W. Paszkowicz, R. Jakiela, B.S. Witkowski, L. Wachnicki, M.T. Klepka, F.J. Luque, D. Arvanitis, J.W. Sobczak, M. Krawczyk, A. Jablonski, W. Stefanowicz, D. Sztenkiel, M. Godlewski, T. Dietl, Phys. Rev. B 88, 085204 (2013)

[50] A. Locatelli, T.O. Mentes, M.A. Ninõ, E. Bauer, Ultramicroscopy 111, 1447 (2011).

[51] A. Locatelli, E. Bauer, J. Phys.: Condens. Matter 20, 093002 (2008) 